Portland State University

PDXScholar

Fall 1-16-2014

\title{
The Small Volume Autonomous Water Sampler (SVAWS): An Innovative, Cost- Effective, Adaptable Protocol
}

Nicholas B. Fitzgerald

Portland State University

Follow this and additional works at: https://pdxscholar.library.pdx.edu/open_access_etds

Part of the Environmental Engineering Commons

Let us know how access to this document benefits you.

\section{Recommended Citation}

Fitzgerald, Nicholas B., "The Small Volume Autonomous Water Sampler (SVAWS): An Innovative, CostEffective, Adaptable Protocol" (2014). Dissertations and Theses. Paper 1533.

https://doi.org/10.15760/etd.1533

This Thesis is brought to you for free and open access. It has been accepted for inclusion in Dissertations and Theses by an authorized administrator of PDXScholar. Please contact us if we can make this document more accessible: pdxscholar@pdx.edu. 
The Small Volume Autonomous Water Sampler (SVAWS): An Innovative, CostEffective, Adaptable Protocol

$$
\text { by }
$$

Nicholas B Fitzgerald

A thesis submitted in partial fulfillment of the requirements for the degree of

Master of Science

in

Civil and Environmental Engineering

Thesis Committee:

James F. Pankow, Chair

Scott Wells

William Asher

Portland State University

2013 


\begin{abstract}
As of 2007 , U.S. pesticide use stood at 1.1 billion pounds with $80 \%$ of all usage in agriculture. Such realities pose a potentially dangerous threat to our surface water bodies. This research provides a detailed analysis of the design, operation and fieldtesting of a prototype water sampler, the Small Volume Autonomous Water Sampler (SVAWS). The research includes a discussion of the design and construction of the SVAWS. Seven USGS employees operated the SVAWS in a number of different states and conditions in order to collect samples for pesticide analysis. Field-testing outcomes of the SVAWS were measured through a Participant Survey Request and a Likert-Scale Assessment.

Overall, survey data proved the SVAWS to be an effective device for collecting water samples in different locations and conditions. Users also understood the SVAWS has significant potential for applications other than pesticide studies. While feedback did show a number of alterations or modifications could improve the design, the device proved to be effective across many of its goals, from valid water sampling to reducing costs of sample acquisition. Recommendations for future research include these and other avenues of research. A conclusion addresses the potential future of the SVAWS with respect to water sampling applications.
\end{abstract}




\section{DEDICATION}

This thesis is dedicated to my mother Jan, my father Terry my sister Kara and my late sister Katie. Without their years of love, nurturing, sacrifice, support, and belief in me, I would not be here today. There has never been a time where they have let me down, and I hope this thesis on a fundamental level reflects the heartfelt gratitude and love I have for them in return. 


\section{ACKNOWLEDGMENTS}

No project of significance is achieved without the guidance, assistance, sacrifice and hard work of others. To this end, I would like to thank Dr. James Pankow, Professor of Chemistry and Civil and Environmental Engineering at Portland State University. His support, knowledge and guidance during this project were invaluable. I would also like to thank Robert Gilliom, Senior Hydrologist, United States Geological Survey (USGS), for developing and coordinating the SVAWS project. In addition, I owe a debt of gratitude to the seven USGS field test members; Chevelle Schreiner, Denis Scanlan, Jeffrey Frey, Megan Shoda, Nathaniel Schaepe, Shannon Stubbs, and Teresa Rasmussen. These professionals not only tested the SVAWS in the field, but they were generous with their time and participated in the Likert Scale Survey.

Senior Research Associates for Dr. Pankow, Lorne Isabelle, Wentai Luo and Bill Asher, were excellent team members that assisted in all phases of the design, development and deployment of the SVAWS project. I could not fail to acknowledge as well, the valuable assistance and knowledge of the local USGS representatives of the Oregon Water Science Center (OWSC), especially the assistance provided by Joseph Rinella, Hank Johnson, and Matt Johnston. Finally, the work of students Hank Chiu, Sam Arnold, Jack Slocum and Joshua Booren advanced the structural design of the SVAWS and allowed for most manufacturing to be done at Portland State University. 


\section{TABLE OF CONTENTS}

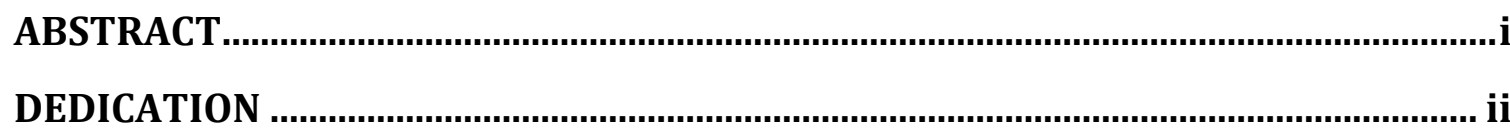

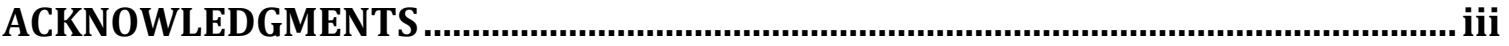

LIST OF FIGURES .................................................................................................... vi

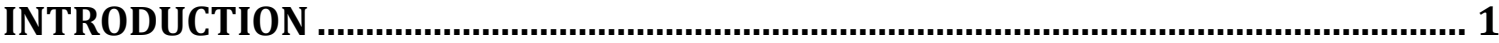

Motivation

Literature Review ..................................................................................................................2

Background ...................................................................................................................................

Research Objectives .......................................................................................................... 9

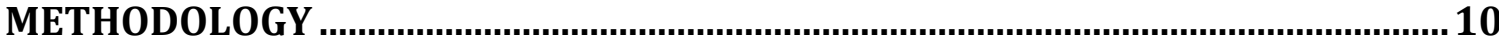

Design and Construction of SVAWS...................................................................... 10

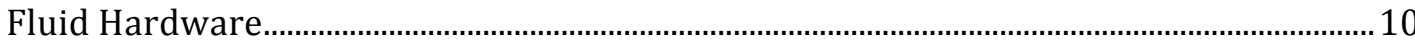

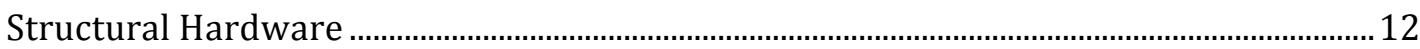

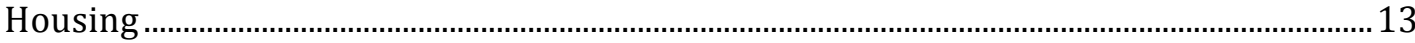

Waterproofing

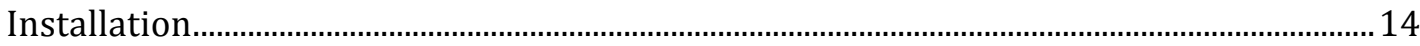

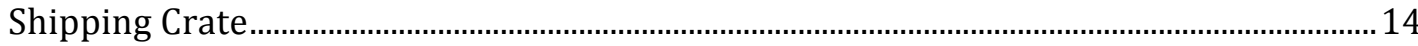

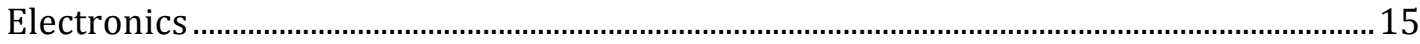

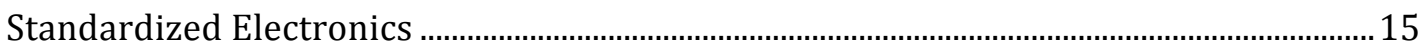

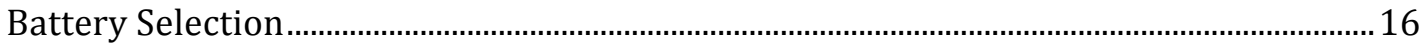

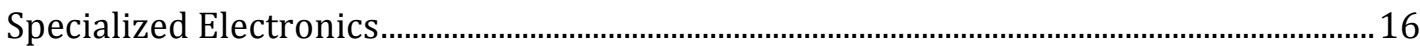

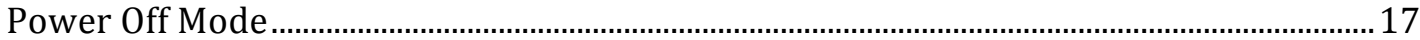

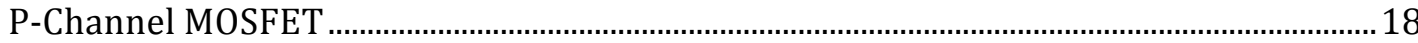

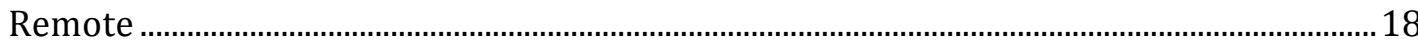

Software and Sample Acquisition...................................................................................... 18

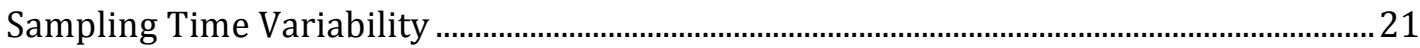

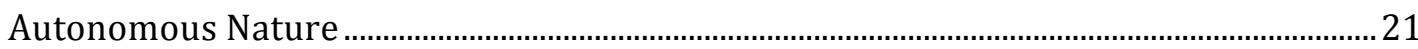

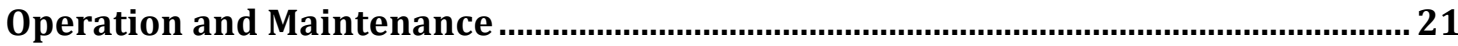

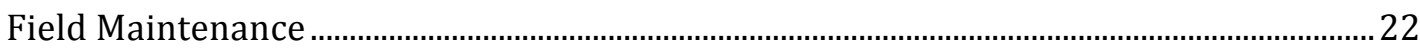

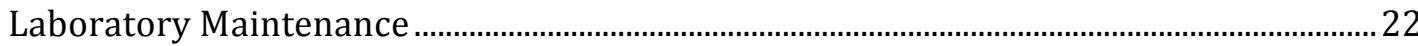

Field Campaign

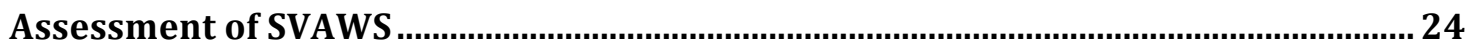

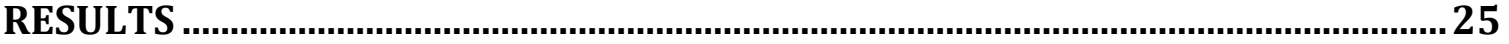

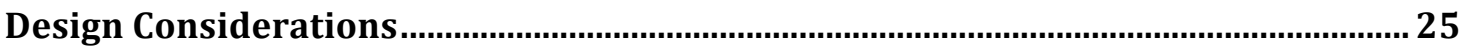

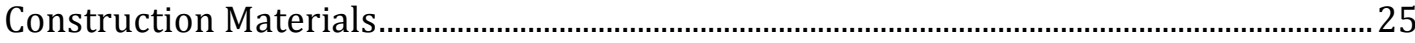

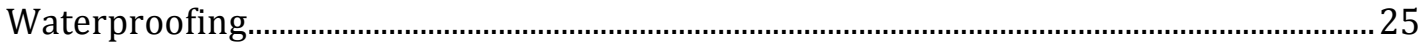

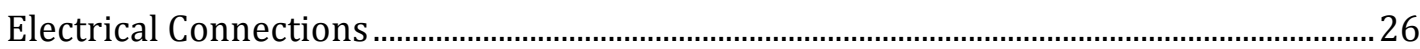




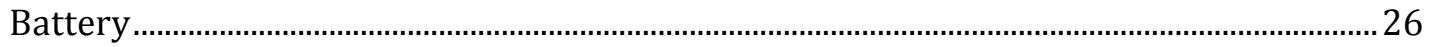

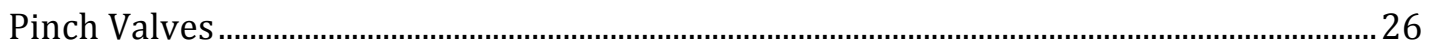

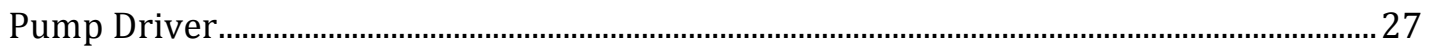

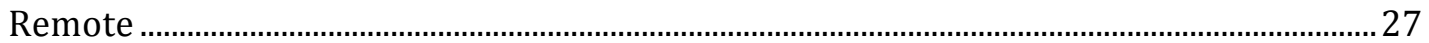

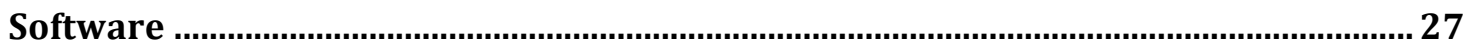

Survey Responses to Essay Questions ................................................................. 31

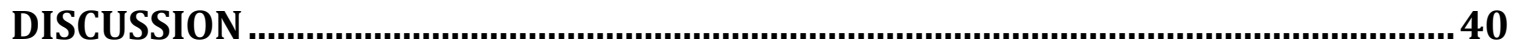

Major Findings of SVAWS Design/Implementation Process ........................................40

Major Findings of SVAWS User Survey ..................................................................... 41

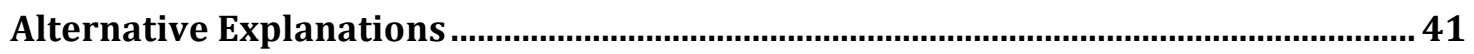

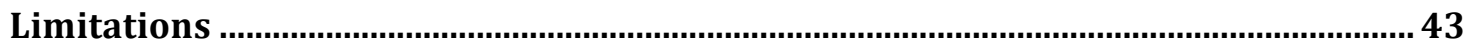

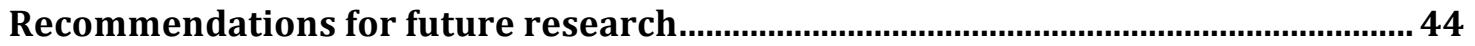

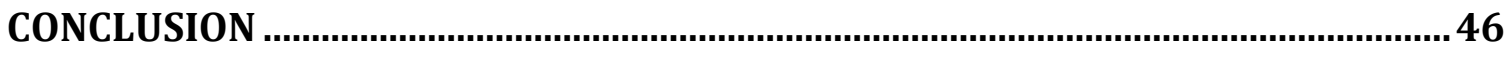

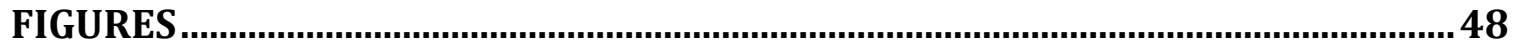

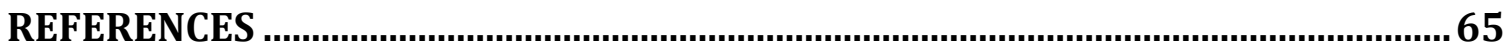

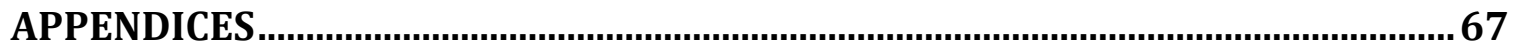

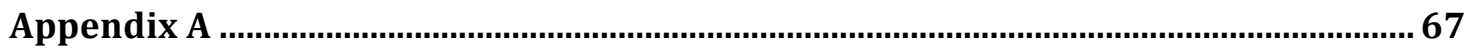

Appendix B

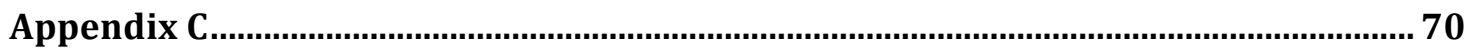

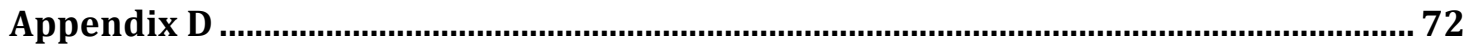




\section{LIST OF FIGURES}

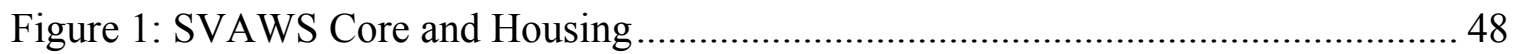

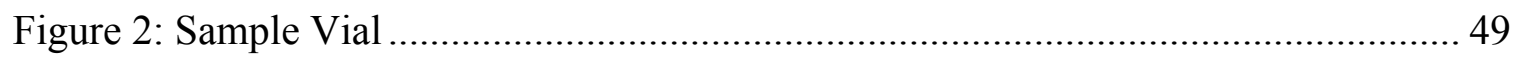

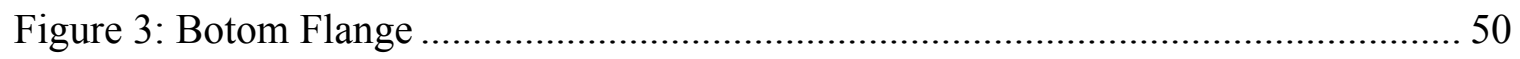

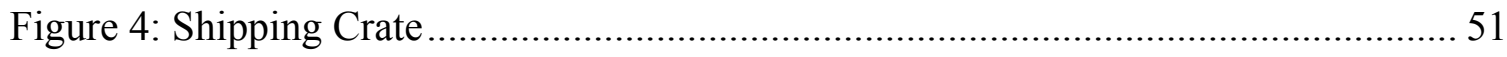

Figure 5: Foam Inserts for Shipping Crate ……………….................................. 52

Figure 6: Electronics Package..................................................................................... 53

Figure 7: Battery ……………………………………………………………. 54

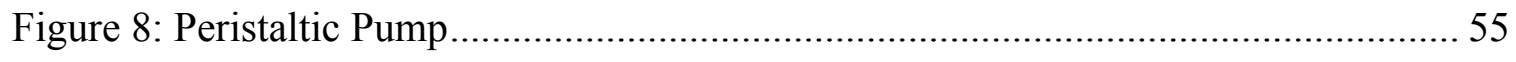

Figure 9: Solenoid Pinch Valve ................................................................................... 56

Figure 10: Overflow Reservoir ........................................................................... 57

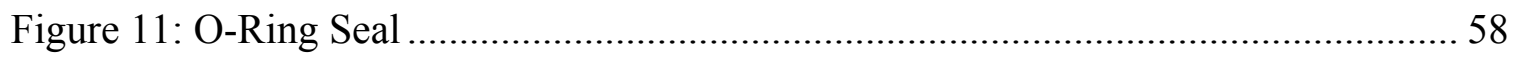

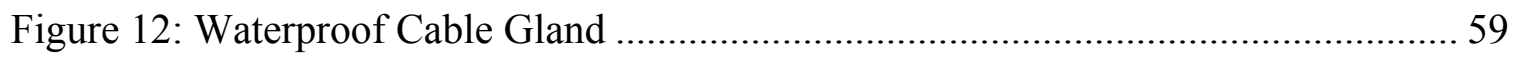

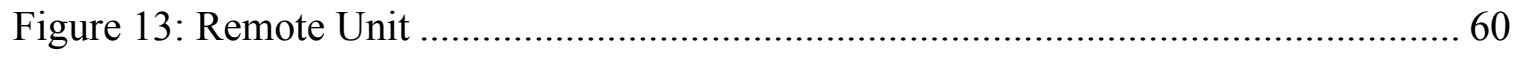

Figure 14: Simplified Flowpath for the Daily Composite .................................................. 61

Figure 15: Simplified Flowpath for the Weekly Composite.............................................. 62

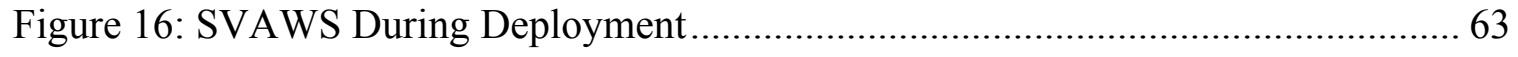

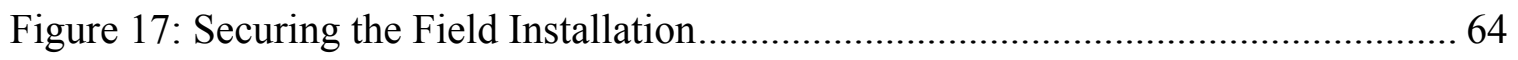




\section{INTRODUCTION}

\section{Motivation}

Pesticide application in agriculture and other industries in the U.S. continues to pose a serious environmental threat for surface water bodies. The latest data available from the Environmental Protection Agency's (EPA's) Pesticides Industry Sales and Usage: 2006 and 2007 Market Estimates (Grube, Donaldson, Kiely, et al., 2011) reveals the following highlights:

- $\quad$ pesticide use in the United States was 1.1 billion pounds in 2007 , or $22 \%$ of the world estimate of 5.2 billion pounds of pesticide use;

- U.S. pesticide sales were approximately $\$ 12.5$ billion at the user level (32\% world market in 2007);

- 857 million pounds of conventional pesticide active ingredient were applied in 2007;

- $80 \%$ of all U.S. pesticide use was in agriculture.

The 2008-09 National Rivers and Streams Assessment (NRSA) produced by the Environmental Protection Agency (EPA) reported:

- $\quad 55.3$ percent of rivers \& streams in the U.S. are in poor biological health.

- 23.2 percent of rivers \& streams in the U.S. are in fair biological health.

- Only 20.7 percent of rivers \& streams in the U.S. are in good biological health.

(EPA, 2010)

Based on the above concerns and realities that are international in scope and have the potential capacity to threaten mankind, the United States Geological Survey 
(USGS) conducted research that focused on the concentration of numerous pesticides used in the agricultural industry on surface water bodies; specifically rivers and streams. Water sampling protocols used in that research are limited in their abilities as they are labor intensive and costly. This research was designed to develop a water sampling method that offers an innovative, cost-effective, alternative protocol of higher efficacy than current USGS water sampling protocols. The SVAWS offers the capacity to meet these needs, in addition to being applicable to a host of other environmentally threatening issues.

\section{Literature Review}

Because the Small Volume Autonomous Water Sampler (SVAWS) is a prototype for a new water sampling protocol, no professional literature exists on this device. The professional literature does offer significant research on more established water sampling protocols routinely employed by the USGS and the EPA. The literature also reveals a strong need for improved water sampling protocols, the purpose of the SVAWS. Since the early 1980s, the USGS (1984) has worked to develop a large data system in collaboration with the states. The objective of the system is to "assure a better balance between water availability and demand" (USGS, 1984, p. 97). This system has yet to be developed primarily due to the "diverse" objectives of the states (USGS, 1984).

The Clean Water Act mandates states provide water condition reports of their waters to the EPA. The EPA uses this data to publish an annual report known as the "National Water Quality Inventory" (NWQI) (Water, 2000). The U.S. Government Accounting Office (GAO) examined various issues of this report. The GAO assessment 
found some primary concerns that reflect a need for improved water sampling protocols. These concerns are: 1) the reliability of data in the NWQI, and 2) whether the data are representative of water quality conditions nationwide (Water, 2000, p. 1). This is a significant issue because state policymakers form key decisions based on the available data.

The increased development of agricultural and urban areas continues to threaten the quality of the U.S. water supply. During the period 1991-2001, the USGS sampled conditions of the nations streams and groundwater to study how farming and development impact water quality (Development, 2004, p. 1). The report found that man made chemical contaminants (e.g. pesticides, nutrients, metals, gasoline-related compounds, and insecticides such as diazinon and malathion) were found in "nearly all the streams" sampled by USGS researchers (Development, 2004, p. 1). This ongoing and increasing threat to the nation's water bodies and water supply demands the most efficient and effective water sampling protocols that provide accurate data.

The need for improved water sampling protocols such as SVAWS stems from the need for policymakers to have informed and accurate data and information when making key decisions related to the health and sustainability of our nation's waterways. Having enhanced data from more effective water sampling protocols is a key issue. So is the need for better coordination of data collection efforts across the nation. To address various issues related to water data collection from various organizations (EPA, USGS, etc.), the Chairman of the Subcommittee on Water Resources and Environment asked the GAO to determine (Stephenson, 2004, p. 1): 
- the key entities that collect water data;

- the types of data they collect;

- how they store the data; and,

- how entities can access the data.

Such concerns show the need for improved accessibility of data in water sampling protocols; features offered by a standardized, automated sampling system such as the SVAWS.

Often controversial water allocation decisions demand accurate and complete water quality data. To this end, federal and state entities charged with collecting water samples are continually seeking new or improved methods of water sampling. The EPA Environmental Technology Verification Program conducted research on two smalldiameter bladder pumps to test for volatile organic compounds, a pneumatic bladder pump and a newly designed mechanical bladder pump (McCall, 2005, p. 142). The EPA research demonstrated that the mechanical bladder pump not requiring compressed air to operate led to two significantly beneficial outcomes:

1) simplification of field logistics; and,

2) reduction in costs of conducting low-flow sampling. These kinds of studies not only reflect the significant benefits that can be recognized from developing improved water sampling protocols, but also that current protocols used by various water sampling entities, including the EPA and USGS, contain room for improvement.

The literature shows another need for water sampling protocols to improve 
them, which is "standardization" and "simplification" of protocols. Hildebrandt, Lacorte and Barcelo (2006) conducted research into the prerequisites for obtaining "reliable" water samples and issues of practical matters for sample collection: planning, field sampling, sampling strategies and equipment, and data quality assessment (p. 1075). The research shows a need for state-of-the-art sampling protocols in order to meet the challenges that currently impact our nation's and world's waterways.

Simplification of sampling protocols is needed, but research conducted by Korfmacher and Musselman (2007) reveals that simplification of protocols is only beneficial "if it could be shown that sample quality is unaffected" (p. 107). Because of this, any newly designed sampling protocol must ensure its benefits are balanced by its capacity to deliver accurate and reliable results. No benefits of improved design are worth sacrificing this paramount need of water sampling protocols. The professional literature clearly shows a pressing need for newly designed water sampling protocols that offer higher accuracy, more reliable data, reduced costs and less cumbersome field logistics. The SVAWS represents an opportunity to meet these needs.

\section{Background}

The USGS developed the National Water Quality Assessment (NAWQA) program to further assess the nation's water condition to determine if the conditions are changing; and, if so, what is driving the change. Over the past few decades the USGS has dedicated significant resources to assess this issue.

In order to make such assessments, the USGS requires water sampling protocols specifically designed to measure analytes of interest. Furthermore, the USGS must test 
hundreds of water samples across multiple locations and often in unscheduled, lastminute situations. The two classes of sampling currently used by the USGS are manual and automated sampling. While both offer benefits, the drawbacks make them labor intensive, cost prohibitive, and demand a more efficacious alternative. This is especially true in light of increasing demands on our world's water supply sources and other bodies of water, which lead to a need for more extensive testing.

One method of manual sampling is known as the "bottle method". The bottle method involves filling pre-cleaned vials in the field with sample water. Water samples are then stored and transported to a laboratory where they are filtered and either frozen or analyzed. This method, although basic, is cost-prohibitive over time when factoring in the costs of field labor. In addition, they also require multiple field visits and staff paid to be on-call if specific runoff events are to be characterized.

The bottle method is implemented for Volatile Organic Compounds (VOCs) following Section of the USGS National Field Manual, as reprinted below:

Samples are collected directly into laboratory supplied prebaked 40 $\mathrm{mL}$ amber vials. While wearing gloves, the sample is collected by uncapping the vial underwater allowing the vial to over fill, then recapping the vial while remaining underwater. It is important to have no air bubbles in the collected sample. Air bubbles can be checked for by shaking vial then inverting the vial to observe if air bubbles are present. If in flowing wadable water it is important to stand downstream of the sample location to prevent contamination.

(USGS, 2010, 4.1.3B)

The description above makes it painfully obvious the potential for human error in this method is high. A crew member might leave bubbles in the vial or inadvertently stand 
upriver while filling a vial. Overall, manual bottle sampling techniques permit a range of sample collection and analytes analysis. However, the need to collect significant and geographically disperse samples, often on the spur of the moment, make this method cost prohibitive.

The second class of sampling is known as automatic sampling. One of the benefits of automatic sampling, unlike manual bottle sampling, is that significantly less labor costs are involved in employing this process. Automatic sampling is also distinct from manual sampling because the processes are categorized into two forms: active and passive automatic sampling. Passive automatic sampling involves placing a device in the environment that collects analytes of interest over the entirety of its deployment. Generally, these inexpensive sampling devices contain a sorbent material that utilizes molecular diffusion to collect the analyte of interest from the fluid phase. Recovery of the analytes sorbed to the sampler can be done with use of extraction or thermal desorption depending on the type of sorbent material. If a liquid collection phase was used, it may be analyzed directly. Analysis of organic samples can be done with gas chromatography/ mass spectrometry (GC/MS) or liquid chromatography/ mass spectrometry (LC/MS). Inorganic samples can be analyzed with ion chromatography (IC), or inductively coupled plasma/mass spectrometry (ICP/MS). Passive sampling drawbacks include a propensity toward being fouled by highly turbid waters and an ability to define only the average analyte level over the duration of deployment. In many cases, it is desirable to know how analyte concentrations changed with time over the sampling period.

Active automatic water sampling is much more cost-effective in terms of labor 
costs when compared with manual bottle sampling. However, with the active automatic water sampling process, capital costs are significantly increased. Active automatic samplers are devices such as the Teledyne Isco sampler that simply automate the proven "bottle method". Generally, these types of samplers use an electronics control system to operate a pump at specified intervals to collect water samples. These can cut recurring sampling costs, as they only require periodic servicing for such tasks as changing batteries and sample vials. These devices contain numerous sample vials and so many samples can be collected and the highly dynamic nature of analyte concentrations can be better studied. Despite these comparable benefits to the bottle method of water sampling, the drawbacks of available active automatic water samplers include:

- $\quad$ high cost per sampler;

- they often employ materials that would interfere with pesticide analysis;

- installation sites can be costly and may require power, concrete \& communication infrastructure; and,

- $\quad$ such samplers are generally non-submersible and are prone to vandalism.

This research will explore an innovative and alternative form of water sampling that overcomes many of the drawbacks of the water sampling protocols currently in use by the USGS, while improving efficacy and lowering costs. SVAWS not only offers the potential for achieving these goals, but it has the potential for multiple applications across diverse environmental issues and multiple industries. This paper will discuss the design, operation, field testing methods, and results of the SVAWS water sampling protocol. 


\section{Research Objectives}

The research goal is twofold. The pros and cons of the design process of the SVAWS will be analyzed, and survey responses will convey the results of field tests conducted by USGS employees to acquire further input on the pros and cons of SVAWS. The innovative design of SVAWS is expressly intended to create a water sampling device that can not only improve on current water sampling methods relied on by USGS, but also to offer scalability for application to other environmental issues.

The objectives of the SVAWS design (See Figure 1) are:

- Simplicity of operation and maintenance.

- Reduced startup and recurring costs.

- Capacity for sampling at many different types of field locations.

- Powered solely by rechargeable battery.

- Low blank levels for a wide range of analytes to provide capability to sample a broad range of chemicals;

- Serve as a platform, allowing for scalability and modification to meet special needs as identified in field research. 


\section{METHODOLOGY}

The methods of designing and constructing the SVAWS included the hardware, electronics, and software. These components were integrated to produce an affordable, autonomous, battery operated, durable, low maintenance sampler that will achieve low blank levels. By selecting common parts and manufacturing methods and by reducing power consumption and minimizing user requirements, these targeted goals were achieved. The methodology consists of three primary elements: the design and construction of the SVAWS, the field-testing of the SVAWS, and the Likert Scale research to assess the effectiveness of the SVAWS in the field. Finally, the development of SVAWS was a team effort. As such, names are listed under each section heading to indicate which teammates assisted in the development of that aspect.

\section{Design and Construction of SVAWS}

The SVAWS is designed for use within a 12" PVC field installation pipe that offers inexpensive sampler protection. The sampler consists of a housing and the sampler core that is designed to float in the water inside the installation. The components required to construct the sampler core and housing are categorized as fluid and structural hardware. Fluid hardware consists of tubing and fittings that can be further categorized as consumable and semi-consumable parts. Fluid hardware greatly influences the ability to achieve low blank levels, a process that will be discussed later along with structural hardware (sampler core, housing and field installation).

\section{Fluid Hardware}


(Team members: Asher, Fitzgerald, Isabelle, Luo, Pankow)

To keep operation costs feasible, the use of consumable parts on the sampler is limited. Consumable parts include:

- 9 25mm Whatman GD/X Filters (Fischer part 6890-2507),

- a three-way check valve (Qosina part 80183),

- $940 \mathrm{ml}$ Volatile Organic Analysis (VOA) vials (Sigma Aldrich part 27382),

- $210 \mathrm{~g}$ desiccant packs (Dry-Packs) and,

- the crimp cap and septa on the waste reservoir

The three-way check valve features an internal diaphragm seal that has the potential for fouling and so requires replacement with each field deployment. The GD/X filters employ a gradated borosilicate bed and membrane to retain particles down to 0.7 um and maintain adequate fluid flow for turbid solutions. The Sample vials feature a Teflon coated septa to preserve sample integrity (See Figure 2). Generic silica gel desiccant packs were used inside the electronics enclosure to reduce the risk of water condensation on critical electrical components.

Semi-consumable parts are those that require only periodic replacement. This category of parts consists of:

- $\quad 0.125 "$ OD Teflon Inlet Tubing (Thermo Scientific part 8050-0125).

- $0.063 "$ OD Teflon Tubing downstream of the filters (Kinesis Scientific part 008T16-100-20).

- $0.025 "$ OD Side Port Needles (Vista dental part 315123).

- Luer Lock Compression Fittings (Qosina parts 80417, 80419, 80428, 80430, 80444). 
The 0.125 " OD inlet tubing was cut to $146 \mathrm{~mm}$ and featured a V-notch on the exterior end to reduce clogging. 0.063 " OD tubing was employed downstream from the Whatman filters so as to reduce the dead volume of the inlet lines. The side port needles are inexpensive and reduce the risk of coring the septa of the sample vials. Luer fittings provide inexpensive, robust and reusable connections between Teflon tubing, filters and needles.

Low blank levels for the analytes of interest were achieved with fluid flow-path design and by thorough washing of all parts prior to assembly. The SVAWS relies on a vacuum driven system so that the inlet lines could be constructed of rigid and inert Teflon tubing. The vacuum system permits pinch valves to be located downstream of the sample vial, so the silicone tubing used in the valves does not contact sample water. To reduce the risk of PVC cement out-gassing affecting sample analysis, PVC welds were used for joining housing components. All sampler parts were washed in liquinox, methanol and, if appropriate, hexane. After multiple rinse cycles with deionized water, washed parts were air dried and assembled in a clean environment by a gloved technician.

A $60 \mathrm{ml}$ overflow reservoir (Nalgene part 2003-0002) was equipped downstream of the pump to contain any water that might flow through the pump in the event of an electronics malfunction (See Figure 10). The HDPE Nalgene bottle was modified with two holes, one for the tubing from the pump and another for a vent.

\section{Structural Hardware}

(Team members: Arnold, Booren, Chiu, Fitzgerald, Isabelle, Luo, Slocum, Pankow) The sampler core consists of the components that mount to the base or interior 
plates. Structural core parts include:

- Standard Pipe to NPT fittings (Swagelock part b-200-1-2) were drilled to create inexpensive bulkhead fittings.

- Structural Rods were manufactured by threading and machining grooves into a 3/8" OD aluminum rod.

- Interior Mounting Plates were either laser-cut from 3/16" thick clear acrylic, or water jetted from 1/4" thick PVC when higher durability was required.

- A Polycarbonate Electronics Case (Otterbox part 3500) with three holes machined into it for mounting waterproof fittings.

\section{Housing}

(Team members: Arnold, Booren, Chiu, Fitzgerald, Isabelle, Luo, Slocum, Pankow)

The SVAWS housing is constructed of a 20 inch-long section of schedule 40 PVC pipe, an 8-inch diameter disk of 1/4 inch-thick PVC for a cap, a 10-inch diameter disk of 1 inch-thick PVC for the bottom flange and four PVC legs welded to the bottom flange. The bottom flange was machined from 1" PVC to allow for sufficient tightening of the 8 bolts around the perimeter. To protect the o-ring mating surface of the flange, four legs were welded to the bottom flange (See Figure 3). This application of PVC provided the following benefits: 1) low cost, 2) durability, 3) ease of machining, 4) heatwelding to avoid glue (as with the cap and flange), 5) it is not expected to be a source for the analytes of interest here.

\section{Waterproofing}

(Team members: Arnold, Asher, Booren, Chiu, Fitzgerald, Isabelle, Luo, Slocum)

Critical components on the SVAWS have three levels of waterproofing as they represent a significant replacement in terms of cost for labor and hardware. The first level 
consists of a 7.725" inner diameter (ID) EPDM O-Ring (Parker part e0603 2-369) used as the primary seal for the sampler housing. This sealed the bottom flange to the sampler base and can be seen in Figure 11. The second level of waterproofing is an enclosure that was modified by adding three waterproof glands, so that category 5 cables could pass through the enclosure and maintain a waterproof seal (See Figure 12). The third level of waterproofing was achieved by adding a generic $10 \mathrm{~g}$ silica desiccant pack to the electronics enclosure to absorb any ambient moisture, thereby lowering the risk of condensation causing electrical shorts. Further, Male RJ-45 connectors were used on the cable ends exterior to the electronics enclosure as these have the potential to maintain a better seal compared to the female ends.

\section{Installation}

(Team members: Arnold, Booren, Chiu, Fitzgerald, Isabelle, Luo, Slocum, Pankow) The SVAWS field installation was built by perforating a 12" PVC pipe, mounting a locking cap to the top end, installing bolts through the bottom end and securing the entire unit at a near vertical angle. To retain pipe strength, perforations were designed to be round and created with a hole-saw. To further increase security of the field installation, the galvanized locking cap is secured from inside the pipe. To ensure the sampler could not exit the installation if a significant drop in water occurred, through bolts at the submerged end of the installation pipe were added. The installation design proved to be inexpensive ( $\$ 300-\$ 500$ for most sites), durable, secure, easy to install and constructed of readily available materials.

\section{Shipping Crate}

(Team members: Isabelle, Luo, Pankow) 
Heavy-duty wooden shipping and transport crates were specially constructed and provided with each SVAWS unit to improve longevity and cleanliness of the samplers. The wooden creates feature a 1/2" plywood exterior stapled onto a frame of 2" by 2 " boards (See Figure 4). A shelf with a round cutout was installed near the bottom of the crate to support the base of the sampler and reduce the risk of the sampler legs being damaged during transport. Exterior hardware consists of 2 handles, 2 hinges, a hasp, and four metal pieces installed on the bottom corners to protect the plywood corners.

Fasteners used for the crates included zinc-plated screws and 2" vinyl coated staples. Five rigid foam pieces were crafted and put in place after the SVAWS was inside the crate, as a way to reduce vibration transfer from the crate to the SVAWS (See Figure 5).

\section{Electronics}

(Team members: Asher, Fitzgerald)

Electronic requirements of the SVAWS were met by utilizing standardized and specialized parts, modifying certain parts, enhancing water resistance, and specifying a battery with future expansion plans in mind. Use of standardized parts lowered costs. Modifications allowed for a strictly battery-operated design, while the increased water resistance and expansion capabilities yielded a more robust design.

\section{Standardized Electronics}

(Team members: Asher, Fitzgerald)

Standardized electrical components were relied on for their low cost and ready availability. These included: RJ-45 and insulation displacement connectors (IDC), cable gland seals, category 5 cable, and 12 volt lead acid batteries. RJ-45 connectors with gold plated contacts reduced assembly time by allowing 8 connections to be made 
simultaneously. IDCs within the electronics package (TE 571-3-640442-2, 571-3640442-4, 571-3-640442-5, and 3m part 3425-6600) reduced labor and material costs (See Figure 6 ). Overall cost was further reduced with use of cable gland seals (Amphenol part LTWCSG-16G) over other forms of waterproof electrical connectors. The 8 amp-hour sealed lead acid battery (Universal Power Group part UB1280) provided enough capacity to significantly increase operation time or run supplemental sensors (See Figure 7).

\section{Battery Selection}

(Team members: Asher, Fitzgerald)

Overall power consumption was reduced so that the SVAWS could remain battery operated and provide capacity for future expansion. A pull-up resistor was added to the R-Drive (the SVAWS microcontroller) so quiescent current of the embedded controller was reduced from $30 \mathrm{~mA}$ to $50 \mathrm{uA}$. A p-channel MOSFET (International Rectifier part IRF9530NPBF) was added to the $+12 \mathrm{~V}$ supply on the pump driver to further reduce quiescent current by $20 \mathrm{~mA}$. The sampler's total power requirement was reduced to 2 amp-hours as a result of these modifications. The 8 amp-hour sealed lead acid battery selected for use (Universal Battery part UB1280) provides ample capacity for routine and hardware expansion.

\section{Specialized Electronics}

(Team members: Asher, Fitzgerald)

Specialized electrical components include:

- the Tern R-Drive embedded controller,

- the Pump Driver (Anaheim Automation part MBC-15081), 
- the Peristaltic Pump (Welco part wpx1-s3/16fa4-c),

- solenoid Pinch Valves (Bio Chem Fluidics part 075p2nc12-02s).

The Tern R-Drive was selected for its onboard high voltage controllers, compact flash memory card slot, analog to digital converters (ADCs), and its ability to power off completely. The peristaltic pump was chosen for its higher level of accuracy $( \pm 3 \%)$ compared to the standard DC pumps Welco offers ( $\pm 7-10 \%)$ (See Figure 8$)$. The Solenoid Pinch Valves were chosen because they offer low power usage (2.9W), are vacuum rated, and the fluid flowing through the valve is isolated from the valve mechanism reducing the potential for contamination (See Figure 9).

\section{Power Off Mode}

(Team member: Fitzgerald)

The Tern R-Drive was modified in order for the onboard real-time clock and a manual override to completely control the device's power status. A $10 \mathrm{k} \Omega$ pull-down resistor connected to the enable pin on the power supply was removed. This was replaced with a $1 \mathrm{M} \Omega$ pull-up resistor. The enable pin was tapped into and shunted to ground with a pushbutton switch, so that the device's power could be manually controlled. These modifications permitted the onboard 3.3V lithium ion battery to provide the quiescent current to control the R-Drive so that no current was drawn from the main battery when the device was powered off. This dropped the quiescent current draw of the R-Drive by $20 \mathrm{~mA}$, and overall power consumption for the sample week was reduced by 3.4 amphours (nearly half the battery's capacity). 


\section{P-Channel MOSFET}

(Team member: Fitzgerald)

An added p-channel MOSFET controlled power to the pump driver and further reduced the weekly power consumption by 1.3 amp-hours. The MOSFET's gate was tied to both $+12 \mathrm{~V}$ via a $10 \mathrm{k} \Omega$ resistor and a high voltage line of the R-Drive. When the RDrive powers down, the MOSFET's gate is held to $+12 \mathrm{~V}$ and no current flows through the pump driver. Conversely, when the R-Drive is active, the high voltage line tied to the MOSFET's gate can be controlled so that the pump driver is powered up. A MOSFET was selected for this application, as it consumes virtually zero current in its powered down state.

\section{Remote}

(Team members: Asher, Fitzgerald)

A remote unit was constructed to allow for control of the SVAWS when the housing was sealed (See Figure 13). In order to expedite the manufacturing process for these remotes, companies were hired for the layout and construction. The remotes feature a 4-Channel dual inline package (DIP) switch, an LED, an RJ-45 connector and a pushbutton interrupt switch. The remote is capable of forcing the SVAWS to power-on, flashing the LED to convey SVAWS output for debugging and sending one of 15 possible codes to the sampler.

\section{Software and Sample Acquisition}

(Team members: Fitzgerald, Luo)

Software design enabled sample acquisition. This was necessary to create an autonomous sampler and vital to the success of the SVAWS. C programming 
language was used primarily, although aspects of $\mathrm{C}++$ were employed when writing to the memory card.

The SVAWS acquires samples by lowering the pressure within a specific sample vial to draw in a sample aliquot. In this manner, the SVAWS collects seven daily composites and one weekly composite during each deployment. The daily composites consist of four aliquots collected every six hours while the weekly composite consists of 14 aliquots collected every 12 hours. Key components to this process are highlighted in Figure 14.

The sampling sequence for a daily composite aliquot begins with a pressure equalization phase, so that the pressure within the correct sample vial is equalized to the pressure inside the sampler housing. To achieve this, the valve connecting the manifold to the desired vial is opened along with the valve that connects the manifold to the interior of the sampler. In this manner, residual pressure in the manifold and vial that may have been present from previous activity is released into the sampler interior.

The next step in the process is the valve connecting the sampler interior to the manifold is shut so that the pump is connected to the current sample vial via the manifold. The pump then activates and draws a vacuum in the sample vial. The reduced pressure in the sample vial induces water flow across the filter and into the sample vial. After the pump runs for a specific period of time, the pump reverses direction and begins to increase the pressure in the current vial to halt the water flowing in. The pump is reversed in this manner to reduce variation in aliquot volumes that were noted in laboratory testing. After pumping in reverse for a specific period of time, the pump is 
then shut off, the valves close and the acquisition of the sample aliquot is complete. At this stage, the sampler either powers down or, if it is time to do so, an aliquot is taken for the weekly composite.

Weekly composite aliquots are obtained with the use of a Teflon sample loop (See Figure 15). The weekly composite sampling process begins when the valve connecting the manifold and the waste reservoir opens. The pump runs to lower the pressure in the waste reservoir. At -1 PSIG, the three-way check valve opens and sample water flows through the filter and inlet lines into the waste reservoir. After a set delay period, the pump is reversed and positive pressure is generated in the waste reservoir. When the waste reservoir reaches +1 PSIG, the three-way valve toggles and the water in the sample loop is pushed into the weekly composite vial. Following another delay, the pump is shut down and residual pressure in the waste reservoir is released to the sampler interior by opening valve 8 . All valves are then closed and sampling is completed.

Following each weekly composite, the sampler runs a back-flushing sequence designed to minimize water intrusion into the inlet lines between sample collection phases. Water intrusion is defined as the slow, unwanted movement of water through inlet lines that can occur as positive pressure in sample vials is lost. Laboratory testing revealed the intrusion process to be relatively slow so the back-flushing sequence is only performed twice daily, after the weekly composite is taken. This back-flushing sequence is performed strictly on the empty vials remaining in the sampler for three reasons:

1. Full vials undergo a full back-flushing after they are filled so that the entire inlet line is cleared of water;

2. Full vials have little headspace so intrusion would not result in unwanted 
water entering the sample vial; and,

3. Back-flushing a partially filled vial created the potential to de-saturate the filter for the inlet line, which could result in variable head loss over the filter and, as a result, highly variable aliquot volumes.

\section{Sampling Time Variability}

(Team members: Fitzgerald, Luo)

Pumping times for daily and weekly composites are reduced as the headspace in the sample vials or the waste reservoir decreases during sampling. The need for this temporal variability was predicted and subsequently verified experimentally by measuring the volume of successive aliquots. Systematically altering pumping times markedly reduced aliquot volume variability.

\section{Autonomous Nature}

(Team members: Fitzgerald)

The autonomy designed into the SVAWS offers the benefits of simplicity, cost efficiency and maximization of the number of potential sampling sites. Use of cellular or wireless communication would increase power requirements and possibly incur monthly data rates. The R-Drive required proprietary software to upload programs, so the samplers were all shipped with the final software versions in place. Because of this, routines were incorporated into the software package for maintenance, troubleshooting and accommodating user error.

\section{Operation and Maintenance}

Maintenance of the SVAWS was minimized where possible to reduce operational costs. To protect electrical components and ensure work quality, 
maintenance was divided into field work and laboratory work. This separation of tasks also reduced cost because the simplified operation required minimal training on the part of the field technicians.

\section{Field Maintenance}

Figures 16 and 17 show the SVAWS during and after field deployment. Upon arrival in the field, a sampler is designed to be deployed as follows:

1. Connecting a remote unit for sending the proper initialization code to the sampler;

2. Lowering the sampler by rope into the field installation tube;

3. Disconnecting the remote unit;

4. Sealing the cable end; and,

5. Locking the installation tube.

Figure 13 shows an operator using the remote unit. Once a sampler has obtained 8 samples, it is replaced in the field with a sampler that was laboratory serviced.

\section{Laboratory Maintenance}

The SVAWS is serviced in a laboratory by using the following 15 -step procedure.

1. The housing is removed.

2. The electrical connections are disconnected and the electronics package is removed.

3. The battery is replaced with the one that is charged.

4. The sample vials are removed and frozen.

5. The waste vial is emptied a new septa is installed.

6. All inlet tubing is connected to the waste vial. 
7. An external vacuum pump is connected to the waste vial.

8. Successive solutions of soapy water, methanol and deionized water are drawn through the inlet lines.

9. The waste reservoir is replaced.

10. Sample vials preloaded with preservative are installed.

11. The three-way check valve and filters are replaced.

12. The electronics package is reinstalled.

13. The SVAWS is reassembled and sealed in the housing.

14. Caps are placed on the ends of the inlet tubes to prevent contamination.

15. The sampler is placed in a secure crate for storage.

\section{Field Campaign}

The seven field users for the SVAWS were assigned to the project by the USGS. Members were distributed throughout the country, with multiple staff in some states including:

- Iowa

- Indiana

- Missouri

- Wisconsin

- Kansas

- Nebraska

The SVAWS units were shipped via specially constructed crates to appropriate USGS offices in the above listed states.

Training information was included in an information packet outlining SVAWS operating instructions and debugging codes (See Appendix A). Five of the field testers 
were flown to Portland State University to receive hands-on training in SVAWS design, operation, deployment and debugging. Personal contact information was provided for additional assistance on any issues related to SVAWS operation or deployment.

USGS staff tested 14 SVAWS units over a four-month period in diverse weather conditions. Samples collected from the testing period were then sent to the National Water Quality Laboratory (NWQL) in Denver, Colorado. The samples were received and frozen by the NWQL for future analysis and testing. Some were combined to form composites for periods of interest.

\section{Assessment of SVAWS}

To test the effectiveness of the objectives of the SVAWS, a Likert Scale Survey was designed along with a Participant Survey Request sent to participating USGS employees via email (See Appendix B). The Likert Scale Survey allowed users to numerically grade SVAWS performance so the device performance could be quantified. Each of the six Likert Scale Survey items quantitatively measured is directly related to one of the primary objectives of the SVAWS design (See Appendix C). Statistical analysis was performed with the Qualtrics.com statistical software package. In addition, the survey also asked for responses to eleven essay questions related to various aspects of SVAWS design and operation (See Appendix D). 


\section{RESULTS}

\section{Design Considerations}

\section{Construction Materials}

Construction materials consisted of PVC housing, acrylic plates, and PVC plates. PVC was used for many of the SVAWS structural components, primarily because of its essentially inert nature with respects to analytes of interest. PVC components were used in place of acrylic, where higher durability was required. Acrylic was selected for use as it is also easily manufactured, readily available, inexpensive and relatively inert.

\section{Waterproofing}

The cable glands and O-ring used for waterproofing the SVAWS were never directly suspected of leaking. Despite this finding, leaks occurred and three samplers were flooded. In one flooding incident, a Qosina fitting was knocked off of an inlet tube during assembly, which effectively created a hole in the bottom of the sampler. This alone does not explain why the sampler filled almost entirely with water. Due to the fact that the sampler filled almost entirely with water; it is believed that air was lost through the remote cable and the water was able to rise much further within the sampler than it would have without the air leak. Correcting the design flaw is imperative, because if a sampler fills more than $75 \%$ with water the valves and pump can be damaged. Replacing the valves and pump costs around $\$ 1000$ when labor, shipping, and parts are considered, so the costs are not insignificant for this failure.

In two instances, inspection revealed that when samplers flooded, a few drops of water travelled through the category 5 cables and were deposited on the R-Drive. In 
anticipation of such an issue, male RJ-45 ends were used outside of the electronics case. However, it is clear from these results an improved alternative is required to seal the cable ends outside the electronics enclosure.

\section{Electrical Connections}

The standardized connectors utilized on the SVAWS included RJ-45s and IDCs. Without exception, the IDC's worked as planned and were the cause of no electrical problems. The RJ-45s also worked as planned, although these connectors needed replacement if they were submerged in water. Three samplers were flooded for various reasons. In each case the RJ-45 connectors needed replacement. Though the need for replacement may be thought of as a drawbacks, the RJ-45s are inexpensive and quickly replaced so the loss in time and capital is minimal.

\section{Battery}

Throughout three months of field trials, no problems with batteries were identified. Although there were some errors that could have been mistaken as faulty battery, it was shown these errors were related to other issues such improper initialization. This determination was arrived at by verifying battery voltage and examining the debug files written to the R-drive's memory card. The debug files were critical to determining if a battery failed, as they contain critical information on when certain phases of sampling were completed. If a battery failed during deployment, certain sampling steps would not be performed and recorded.

\section{Pinch Valves}

All but one of the 131 valves utilized in the field campaign worked as expected. 
It is worth noting that there were 36 valves submerged in river water for up to a week at a time when four samplers were flooded in the field. When RJ-45 ends were replaced and the valves were tested, many appeared to function normally. The valves that were submerged however were not reused due to the risk of malfunctioning.

\section{Pump Driver}

The microstep driver needed to operate the pump was never found to be faulty nor was one suspected of failure. Any one of the multiple debug codes that utilized the pump could be used during lab maintenance to verify operation of the pump and pump driver. Further, it can be assumed that the p-channel MOSFET used to power the pump driver worked as this component allows for driver and pump function.

\section{Remote}

The remote for the SVAWS system permits samplers to be sealed at all times following laboratory maintenance, provided standard deployment techniques are employed. The remotes gave field personnel rapid, remote determinations of system needs and allow the user to initialize various routines on a sealed sampler. Throughout the field campaign period, there was no reported instance of remote failure.

\section{Software}

Throughout the three-month field tests the software written for the SVAWS showed no signs of failure. In a majority of instances the SVAWS initiated properly in sample collection. Although the number of sampling routines was limited, the package provided adequate means for USGS requirements. Midway through the field deployment campaign, the USGS needed to utilize a different initialization sequence. The available 
software loaded to the device provided an alternative solution that addressed the new requirements. 


\section{Likert Scale Survey Responses}

Results from the survey of the 7 SVAWS users are given below. The entirety of these survey results may be found in Appendix C. Note that the first 6 questions were Likert Scale type questions while the last 11 were text response.

\section{Likert Scale Q.1: How difficult was operation of the SVAWS sampler?}

Of seven respondents, the average value for difficulty of operation of the SVAWS sampler was $63.57 \%$, with standard deviation of $23.22 \%$. With zero percent being "very difficult" and $100.00 \%$ being "very simple", a majority of the respondents tended to view SVAWS operation as above average in ease of operation. With a minimal value of $30.00 \%$, no respondent felt SVAWS operation was "very difficult".

\section{Likert Scale Q.2: How difficult was maintenance of the SVAWS sampler?}

On a scale with zero percent being "very difficult" and 100.00\% being "very simple," difficulty of maintenance for the SVAWS yielded $51.20 \%$. Of the seven respondents, the minimum value was $9.00 \%$ and the maximum value was $92.00 \%$. Despite these extremes, standard deviation for all data was $29.26 \%$. The fact that someone felt the SVAWS was close to "very difficult" to operate and one other felt it was close to "very simple," could be accounted for by personal viewpoint of maintenance and effort required. SVAWS maintenance proved, on average, to be relatively simple.

Likert Scale Q.3: Based on your experience, do you believe the SVAWS sampler is more or less costly to install and operate than other automated samplers (such as 


\section{those made by Teledyne Isco)?}

Only six of the seven respondents provided data for this survey item. Of those respondents, the average value for whether or not the SVAWS was "much more costly" $(0.00 \%)$ or "much less costly" (100.00\%) than other samplers is $59.83 \%$. This reveals that testers in general believed the SVAWS to be less costly than comparable water samplers. However, the minimum value of $9.00 \%$ was recorded in addition to a maximum value of $100.00 \%$.

\section{Likert Scale Q.4: Does the design of the field installation and SVAWS sampler allow for installation at many or few field sites? IE are many or few available for sampling?}

Of the seven respondents for this survey item, average value was $49.00 \%$, with a standard deviation of $34.60 \%$. With "few sites" defined as $0.00 \%$ and "many sites" defined as $100.00 \%$, one respondent posted a minimum value of $10.00 \%$, compared to another that posted a maximum value of $100.00 \%$. In general the SVAWS sampler falls in the mid-range from deployable at "few" or "many" sites. However, it seems such a wide ranging minimum and maximum value could be related to geographical conditions in addition to availability of nearby suitable sites.

\section{Likert Scale Q.5: How many times did you note that a fully charged battery onboard the SVAWS discharged completely during a one week field deployment?}

Battery discharged was marked on a scale from 0 to 20 times. Only four respondents out of seven reported on this survey item and all recorded values were scores 
of 0 . These statistics reveal that the SVAWS battery did not fully discharge during any of the one-week deployments.

\section{Likert Scale Q.6: Do you believe the SVAWS sampler has the potential to serve as a platform and be used for various future applications?}

To measure whether the SVAWS sampler has the potential to serve as a platform and for future applications, $0.00 \%$ was ranked as "Definitely Not," while $100.00 \%$ was "Absolutely". Positive response for SVAWS as a potential platform and for use for future applications is reflected by an average value of $73.43 \%$. Also positive in this regard is the fact that this item received the highest mark for a minimum value in the survey, $49.00 \%$, in addition to more than one maximum value of $100.00 \%$ ("Absolutely"). Further, this survey item showed the lowest standard deviation of any of the items, except for battery discharge, at $21.54 \%$.

\section{Survey Responses to Essay Questions}

For all comments collected for the essay questions of the survey, see Appendix D.

\section{What do you think would simplify the operating procedure of the SVAWS sampler?}

Of the 6 respondents who answered this question, most felt the SVAWS was fairly simple to operate. One respondent commented; "I can't think of anything that would simplify the operating procedure of the sampler". Another respondent maintained: "The actual operating procedure is pretty easy - take the sampler out of the box, put it in the PVC tube, press start command". Despite such comments overall, there were concerns and issues raised that will help improve future models of the SVAWS. One 
respondent felt supplying respondents with more detailed installation instructions would have been useful, while another's biggest operational concerned stemmed from fears that they might return to the sampler site a week after deployment and discover nothing had happened.

One response that would make operation more efficient and less cumbersome involves replacing the nuts and bolts with a form of watertight closure that does not require tools or as much effort to open and close the SVAWS. Another suggestion useful for possible inclusion in future models of the SVAWS is a digital controller with built-in commands, such as "clean cycle," "begin weekly sampling," etc.. Such features were not overlooked in the design of SVAWS but are absent in the prototype due to budget restrictions.

\section{What do you think would simplify maintenance of the SVAWS sampler?}

All seven respondents returned comments on this aspect of SVAWS. There were numerous suggestions regarding the maintenance of SVAWS. These ranged from operational to pre- and post- deployment instructions and training. Operationally, one of the biggest issues stemmed from the use of the SVAWS category 5 remote cable. In Iowa, the cable caused multiple issues. The original cable was too short and so a replacement needed to be installed. The cable also had the potential to slip through the waterproof gland when tension was applied. Another user stated the outer sheathing of the cable was stripped away by the constant movement of the sampler. Both comments imply that at some points the cable might bear the weight of the sampler. A cordless sampler could resolve this issue. 
Other recommendations included expanding the test code to indicate whether "adequate vacuum" is occurring in vials. One user discovered a single sampler had trouble filling vials 5 and 6 but did not recognize the problem until the end of a week when the sampler was serviced. Other comments addressed weekly composites that did not sample for a given week, with little explanation or reasons why. SVAWS also proved to be susceptible to connections that loosen if not handled delicately during deployment.

Where instruction and further training are concerned, one respondent suggested a more detailed manual with "step-by-step" operating instructions. This could include a trouble-shooting section where common problems with suggested corrections are provided. One SVAWS respondent suggestion that additional training would be helpful, including "expected performance". Such information and more thorough training would alleviate many of the issues experiences with maintenance of SVAWS.

\section{What changes to the SVAWS sampler would lower operating costs?}

Only 4 respondents commented on this suggesting the others did not recognize many changes to lower operating costs. Of those that did, the most common observation was that the "sampler should be able to be lifted, cleaned, and prepared by one technician". Another method of lowering operating costs would be to reduce the amount of time it takes to clean the sampler (35-40 minutes) before deployment. This maintenance also required 2 technicians at various stages.

One respondent thought costs might be further lowered by eliminating some of the fittings associated with each sample line. Time is spent "checking and rechecking each and every sample line" for pressure leaks, one respondent wrote. A solution to 
identify and address vacuum leaks could come in the form of a mock sampling sequence to be preformed with the housing removed. Not all respondents felt the SVAWS design could be altered to minimize costs. One explained: "Actual operating costs (supplies, etc.) seem pretty low. Best thing is to simplify cleaning and prep to reduce maintenance time".

\section{What changes would reduce costs of the SVAWS field installation?}

Where reducing costs of SVAWS field installation is concerned, 5 respondents commented. Many of the comments were positive. One of the respondents commented: "I don't have any suggestions on how to reduce costs for the field installation of the samplers. It is pretty easy to install the PVC tubing and the sampler". Another stated that "Field installation was relatively inexpensive", although the "most difficult aspect of installation was maneuvering the long 12" PVC tube". In addition, for some site installations the PVC needed to be much longer than for others, so it made the connection "slightly unstable". Zionsville had the most costly installation, where 30 feet of pipe, weighing about 300 pounds, required 4 technicians to vertically install it off a bridge. For future prototypes of the SVAWS, one of the respondents suggested a "floating" sampler that would not require such an elaborate setup, especially where natural or manmade obstacles exist such as high bridges or steep banks.

\section{What changes could be made to the SVAWS system so that more field sites could be accessed?}

Five respondents commented on this aspect of the SVAWS design and operation. One respondent felt the SVAWS is ideal for testing at multiple field sites: "I can't think of any changes to make more field sites accessible. The installation is easy 
and the samplers are self-contained units so they should be fairly easy to install and use anywhere". One respondent felt site selection and installation were "problematic" due to a number of issues. One observation was that in its present design, it would be impractical to deploy the SVAWS at a site with low, gradual banks and no bridge. One respondent suggested a "bank-anchored standpipe" could be a modified install to make the SVAWS functional in such an environment.

Other comments illustrated SVAWS may not be suitable or may need structural modification to work effectively in some environments. One user explained: "The SVAWS was not conducive to Nebraska's mud-bottom and high sediment level streams. The sample tubing would often be obstructed, not allowing a proper sample to be collected". Such an observation suggests that the bolts at the bottom of the installation pipe might be raised so that the sampler is prohibited from sinking to the point where its inlet lines contact river sediments.

\section{What other studies can you envision performing with the SVAWS system?}

One respondent suggested the SVAWS might be ideal for nitrate sampling, while another argued the SVAWS is useful for "just about any study that can use $40 \mathrm{ml}$ or less of sample volume for the testing". One user maintained that if SVAWS data are "proven reliable," then the sampler would be perfect for "studying other organic determinants such as wastewater compounds and pharmaceuticals". The same respondent suggested this would be especially of interest in urban areas "looking at fate and transport runoff and in wastewater discharges".

Despite such positive commentary, one respondent to the survey found few 
other application for the SVAWS: "The idea of the sampler is a great one, but I would not recommend using the sampler for any other studies at this time”. Such comments were however, in the minority. In general an overwhelming majority of respondents saw multiple application of the SVAWS to other studies.

\section{What would you change about the SVAWS system?}

Six of the 7 respondents provided comments or recommended changes regarding the SVAWS system. Chief among the recommended changes are:

- More detailed data sharing (volumes of sample, which bottles aren't filling, etc.).

- Encase all electronics in waterproof boxes.

- A more "user-friendly" remote of higher durability.

- A higher quality remote cable or wireless operation.

- Limit the number of fluid connections.

- Better attach the legs to the bottom of the sampler.

- Include a command to sample immediately in each of the vials.

- Create a feedback system that makes it easier to know when and why some samples are not being collected.

Other than these changes, respondents noted few issues related to the SVAWS system. One maintained that "Due to the large quantity of small parts, there are many places that air can be let into the system. If any of the couplers or tubes were to come loose, the entire sample is affected". One respondent explained the inside of the sampler "was at a much higher temperature than the water". High internal temperatures could certainly impact analyte degradation rates and should be investigated. 


\section{What did you like about the SVAWS system?}

Receiving a response from all 7 users, the SVAWS features aspects liked by all users. One simply liked the SVAWS for its unique concept. Another appreciated "the idea of it...I think it's a really great idea and I really want to see it become a viable sampling option". The SVAWS worked well enough that it helped overcome the initial doubts of one user who maintained: "I was a little skeptical at first, but I was impressed overall with how consistently the system worked".

The "portability" and its "relatively easy installation" were other qualities of the SVAWS system liked by users. The ease of field deployment was corroborated by another respondent: "I like the simplicity of the field procedure...easy to hookup, easy to turn, and all done (if everything worked right)". In contrast to some respondents who found the SVAWS laborious to maintain, one respondent maintained "I like cleaning in the lab/office and swapping out complete samplers during each field visit". Another respondent commented on the backup features of the SVAWS: "I liked having backup systems so they could be maintained in the office and then exchanged in the field".

\section{What didn't you like about the SVAWS system?}

All 7 respondents commented on aspects of the SVAWS system they did not like. Most of these dislikes have been commented upon in previous sections of the survey, such as the number of fittings and the small tubing. Another referred to the number of "small pieces" that can be easily lost and make the SVAWS more difficult to assemble once it has been taken apart. The need for "better training" for staff was also 
referenced.

There were some dislikes among respondents not previously discussed in the survey. One disliked the "inconsistent sample volumes" collected by the SVAWS, and one felt the top-positioned and heavy battery should be replaced by a "smaller power source" if possible. Another respondent disliked the fact that the SVAWS was prone to breaking at times, "from a leg falling off" or a "plate breaking" to vials "overfilling". The positive aspect of these comments is that most of these dislikes are relatively simple or inexpensive to alter or fix on future models of the SVAWS, I.E. using a smaller power source and more secure legs on the base. Such feedback is critical for designing a superior SVAWS system in future prototypes.

\section{If your SVAWS ever flooded, what was the cause of the flooding? Do you have any comments related to SVAWS flooding?}

Of the 7 respondents who commented on this aspect of the SVAWS, one respondent stated outright: "Our samplers were never flooded". While one respondent maintained the samplers did not flood, they did report occasional water in the sampler due to "overfilling" of vials. Another respondent stated that the sampler only flooded "once," but the tester was unsure of the reason. The same respondent speculated that the flooding might be due to "a large runoff event" experienced that week.

A final respondent remarked that one sampler flooded once, but the issue seemed to arise from "leaky connections between the inlet tubes". The same respondent felt the seal of the container should be modified to ensure water cannot leak into the body of the sampler. Overall, flooding did not seem to be a common or major issue, but this 
feedback suggests ways to help eliminate flooding altogether, even in severe conditions.

\section{Final Comments?}

Five of the field testers provided final comments on the SVAWS system. One felt the experience was ideal: "Overall, I loved the Pankow" (aka SVAWS). The only recommendation from this field member was to reduce the number of connections (tubing and electrical) that offer the potential for failure. The same member also expressed future interest in the SVAWS: "I would definitely be interested in knowing how the product is changed and also in working with it again in the future". One field tester was so pleased with the actual performance of the SVAWS they overcame initial doubts and suspicions regarding operation:

I predicted that the filters would clog with sediment during runoff events that could have caused pressure problems with lines popping off, etc. I was pleased to discover that never happened, which makes me more optimistic that the sampler is useful in high turbidity deployments.

Another respondent offered "video" training as an effective tool for future field tests, while another maintained the development and support team offered a high level of customer service: "I thought the Pankow development and support team were responsive to problems and provided timely and useful suggestions to address our problems, so that was appreciated".

One negative comment came from a tester who felt the SVAWS needs much more field testing. This user had difficulties in part because of the site location, which required suspending the device 30 feet from a bridge. However, the user felt training was 
inadequate, stating: "At the training in Portland, we didn't actually get to see a working sampler in action. It almost feels that the USGS was the 'guinea pig' or 'beta testers' for this sampler". While appreciating the hard work and resource put in SVAWS, this respondent did not think the sampler is a "good fit" for the project undertaken. Such feelings were isolated to this one respondent. The difficult installation and site conditions may have lead to such an impression of the SVAWS system.

\section{DISCUSSION}

\section{Major Findings of SVAWS Design/Implementation Process}

An analysis of the from the SVAWS design process reveal these significant findings:

- The remote cable proves highly vulnerable to costly air leaks and needs modification.

- While not as costly as remote cable air leaks, infrequent but potentially significant water leaks through device control cables needs corrected.

- Unless flooding rises past the connector level, corrosion of the RJ-45 connectors was not detected.

- The plunger of the pinch valves is susceptible to flooding, but only if remote cable air leaks permit water levels to reach the valves.

- Throughout three months of field trials, there were no reports of battery failure.

- There were no failures reported of the pump driver.

- No bugs were identified in the SVAWS software during the 3-month field test.

- The cable glands and O-ring used for waterproofing the SVAWS were never suspected of failure. 


\section{Major Findings of SVAWS User Survey}

The findings of the SVAWS field tester surveys revealed a number of positive and negative aspects of SVAWS design. Analysis of the survey data highlights these significant findings:

- Testers liked the portability and easy installation of the SVAWS.

- The SVAWS housing proved waterproof in the vast majority of deployments.

- SVAWS proved useful for testing areas with high turbidity deployments.

- SVAWS proved useful for operating effectively during large runoff events.

- User inexperience with SVAWS implementation revealed significantly lower levels of sought sample collection during the first 9 weeks.

- More detailed data sharing is required.

- A more durable and lighter weight design is needed.

- The number of small pieces, moving parts and connections needs significant reduction.

- A more user-friendly remote is warranted.

\section{Alternative Explanations}

There is significant relevance to the potential alternative explanations for a number of features or operational aspects of the SVAWS that survey results have shown may benefit from design improvements. This encompasses a number of issues, such as the SVAWS not taking samples or filters clogging.

\section{No Daily Composite Taken}

- Improper sampler initialization 
- RJ45s connected improperly

- Manifold leak

- Pump wires disconnected

- Driver wires disconnected

- Inlet lines bedded in river sediments

No Weekly Composite Taken

- Fittings for sample loop leaking

- Check valve installed incorrectly

- Poor tolerances for the cracking pressure of the three way valve

\section{Filter Clogging}

- Inlet lines bedding in river sediments

- Fluid connection failure in the field midway through sampling could have been interpreted as filter clogging

\section{Inconsistent Sample Volumes}

- Filter head loss variations

- Small leaks in fluid fittings

- Use of Luer filters, instead of the specified Luer-lock filters

\section{Vials Overfilling}

- Driver wire disconnection

- Samplers trapped under water instead of floating

- Variations in the dead volume in the manifold and pump tubing

The alternative explanations listed above are considerations worth investigation to help mitigate issues most noted by USGS staff. Knowledge of these potential explanations may help users improve their capacity to install and operate the SVAWS in 
future tests.

\section{Limitations}

There are various limitations associated with the research. The most obvious of these is the small sample population used to test the SVAWS. Only 7 users were utilized in the field trial, and not all of them provided feedback on all survey questions. Another limitation is the lack of varied conditions and water bodies in which the SVAWS was tested. Harsher conditions or alternative water body environments (sandy, rocky, muddy, etc.) would provide a broader array of feedback related to the suitable of SVAWS in different conditions and environments.

Another significant limitation in the study is limited resources. Budget concerns and considerations led to the choice of design materials and overall construction and operation of the SVAWS system. For example, the PVC pipe was the most suitable for the task at a cost that was within budget. Many complained of its cumbersome nature for installations that required lengthier pieces of PVC pipe. Limited resources also precluded some suggestions made by testers, such as a wireless SVAWS device, more detailed and remote data, and a more durable construction. Cost and power issues are also factors in why the SVAWS is lacking refrigeration, which would reduce analyte degradation.

A possible limitation that may have skewed some results or undermined the reliability of samples is the assumption the river's cross-section is well-mixed. Since testing was only conducted at a single point in each river, this may not be the case and water content at other points in the river might vary from data retrieved. Another limitation is that sampling occurred at set intervals. Due to this, the sampler was 
unable to take samples at other times when conditions varied and might be ideal for testing, such as during high runoff events. Lack of time to do operational tests with the trainees and the fact that all trainees could not attend the Portland training session were also limitations, especially in light of a significant number of respondents wishing training included actual deployment and operation of SVAWS in addition to more detailed training.

\section{Recommendations for future research}

Future research should aim to address some of the limitations of this project. This includes sampling a larger number of water bodies in more diverse conditions. Future research should also include modifications to the SVAWS as reflected in the feedback analyzed herein. For this reason, the following recommendations are made for an evolved SVAWS prototype that will overcome many obstacles when tested in future research studies:

- Add sensors to make the SVAWS sample collection event-triggered.

- Adjust sample acquisition routine so composites with more aliquots can be acquired.

- Add a system of refrigeration to limit analyte degradation.

- Develop housing modifications that provides faster access to the sampler core.

- Identify a valve option for the SVAWS that is less costly.

- Develop more debug codes for the SVAWS remote for lab controlled changes.

- Wrap the installation pipe in mesh or bore smaller holes so large debris can not enter the pipe.

- Develop an installation for the SVAWS designed for high bridges. 
- Analyze biofouling of the samplers over time.

- Adapt the system to other controllers such as the Arduino platform to reduce sampler cost.

With the recommended alterations and modification of the SVAWS noted above, future research into these other areas and aspects of operation will reveal much greater information and insight into the suitability, feasibility and effectiveness of using the SVAWS sampler to effectively collect water samples for analysis. 


\section{CONCLUSION}

In conclusion, the goals of this research were to analyze the pros and cons of the SVAWS design process and to assess survey responses from USGS employees to determine the feasibility, effectiveness and suitability of the water sampler. Overall, the SVAWS proved itself to be reliable, easy to install, lower in cost than other types of water samplers, and effective in collecting multiple water samples. While a number of issues and design concerns were noted by USGS users, a majority of them felt that the SVAWS preformed its mission and holds the potential for numerous applications in the field in future.

The continued use of pesticides and other water pollutants in society demands a water sampler that is cost-efficient, effective, and able to collect water samples in diverse locations and conditions. The SVAWS prototype seems well-suited to meet these challenges. Based on this research, future models will require some changes in design, installation and operation. With such changes made, the SVAWS has promise for use across multiple applications and install challenges, including high turbidity areas or other conditions such as high bridge installs. The SVAWS also offers scalability for application to other environmental issues.

Research on the SVAWS has revealed its innovative design yields the possibility for its use by professionals over other more costly, cumbersome and/or less effective water samplers. Additional testing is required to pinpoint certain design and operation aspects of the SVAWS that may require modification, if they are not due to 
some alternative explanation. Research shows there is a desire among professionals to have a means of water sampling that overcomes such challenges as these, in addition to providing scalability.

In summary, the SVAWS was designed to create a water sampling device that improves upon current water sampling methods relied on by the USGS. This is true in terms of ease of installation, cost, sample reliability and operation. This research demonstrates that with simple modifications and alterations, the Small Volume Autonomous Water Sampler meets these needs, in addition to being applicable for use in other potentially threatening issues aside from pesticide sampling. With over half of the rivers and streams in the U.S. in poor biological health, there is a strong need for improved water sampling devices by the USGS and other environmental organizations, agencies and advocacy groups. This bodes well for the future use of the SVAWS, especially once evolved prototypes are designed with the information acquired during this research project. 


\section{FIGURES}

Figure 1: SVAWS Core and Housing

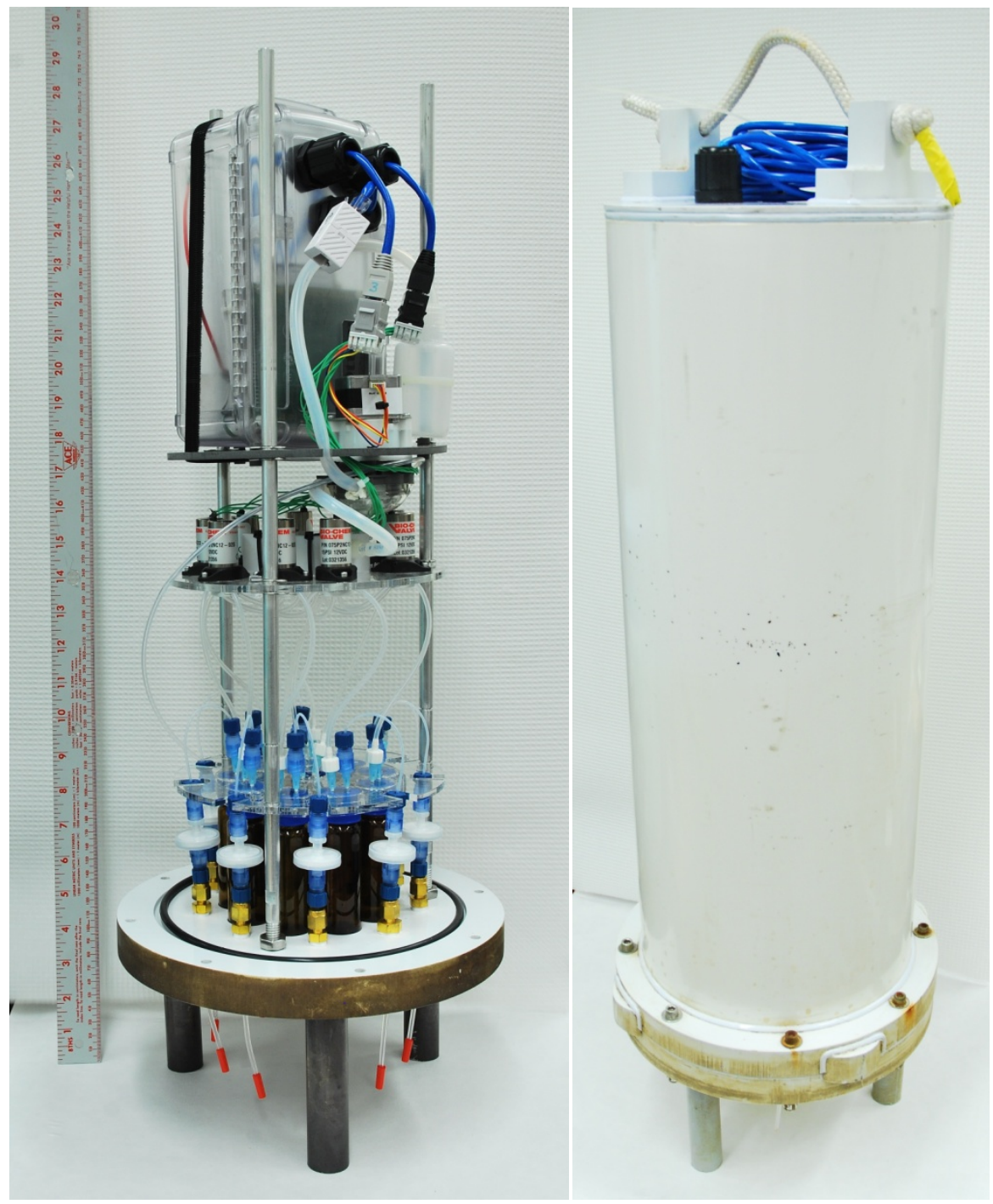


Figure 2: Sample Vial

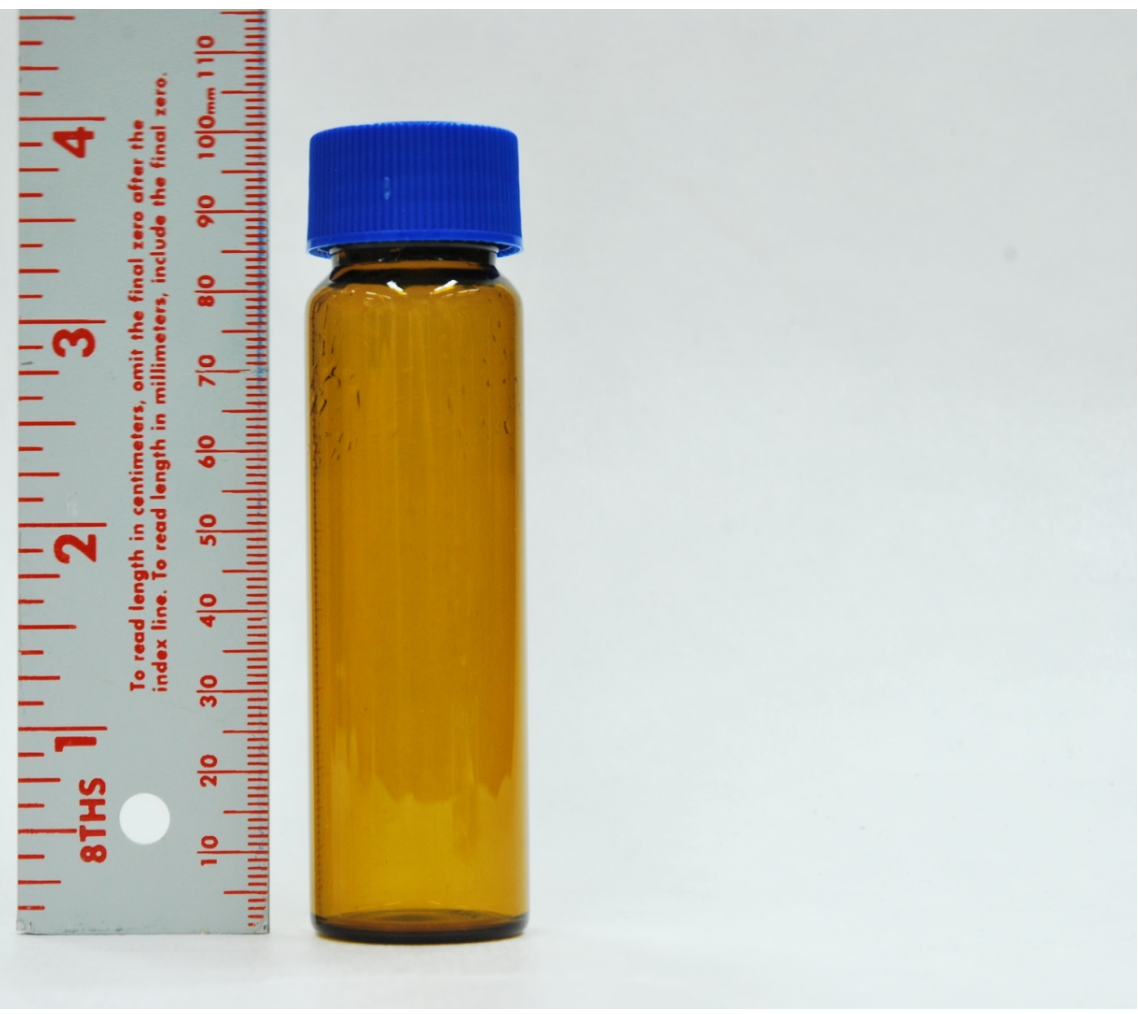


Figure 3: Botom Flange

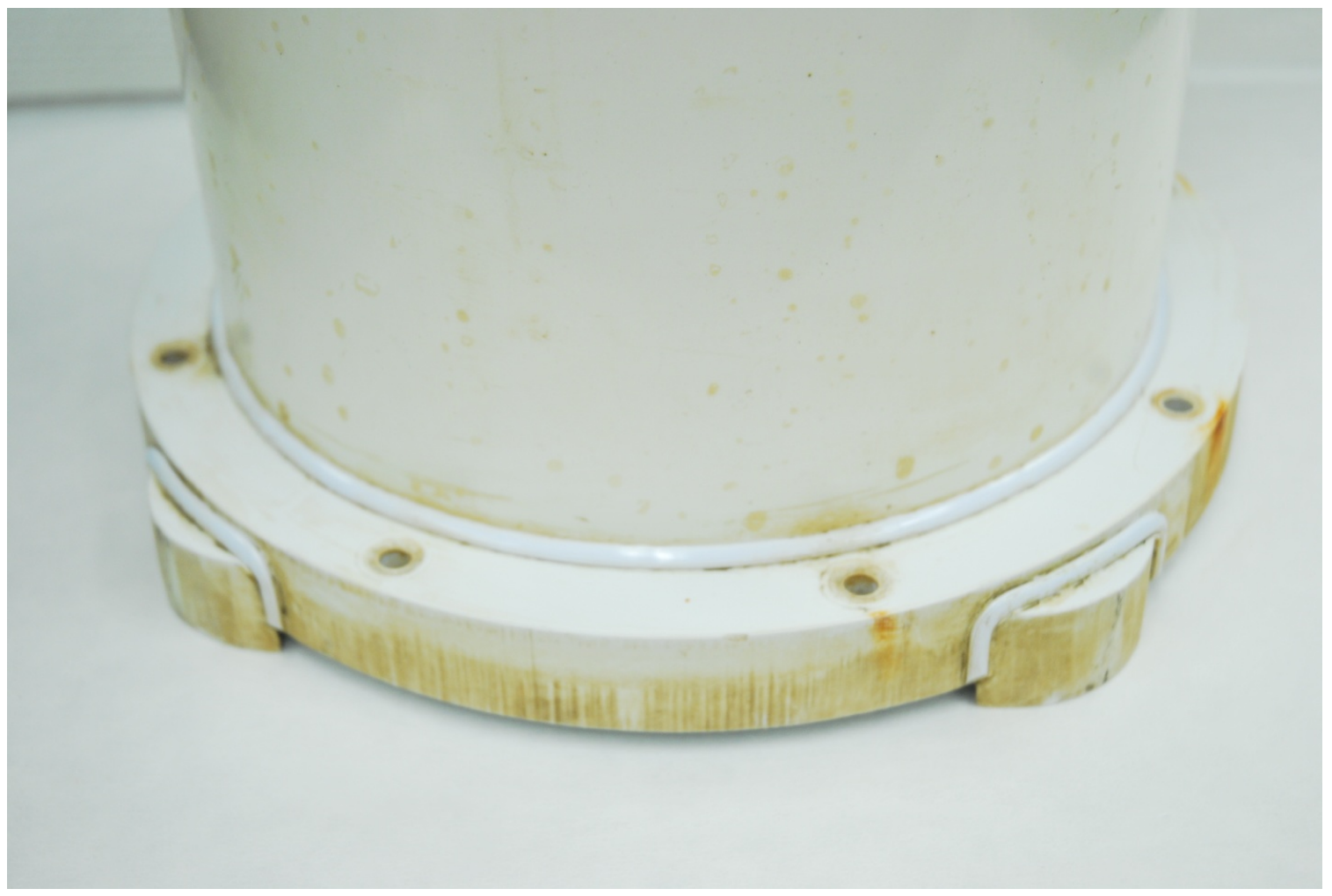


Figure 4: Shipping Crate

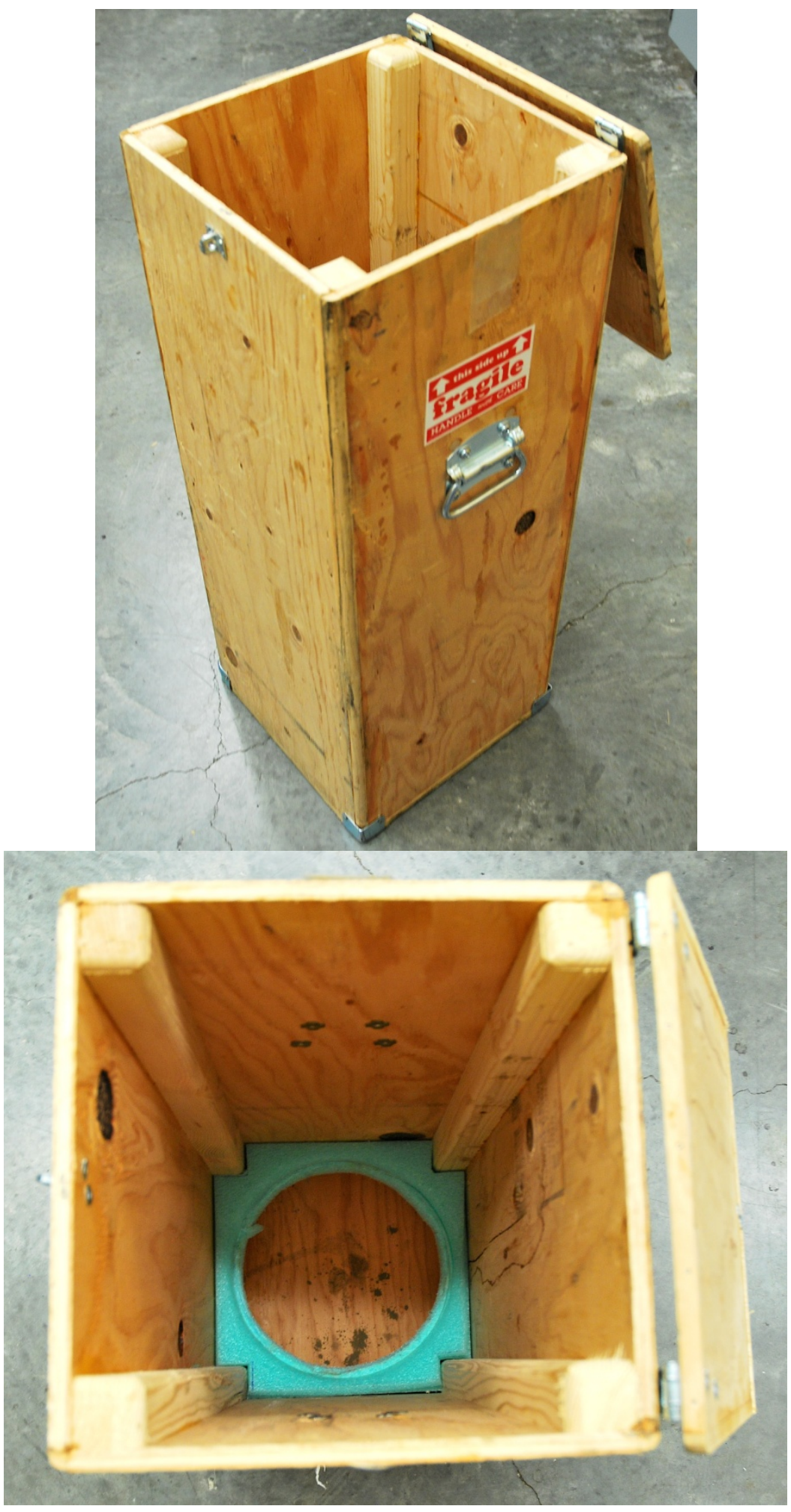


Figure 5: Foam Inserts for Shipping Crate

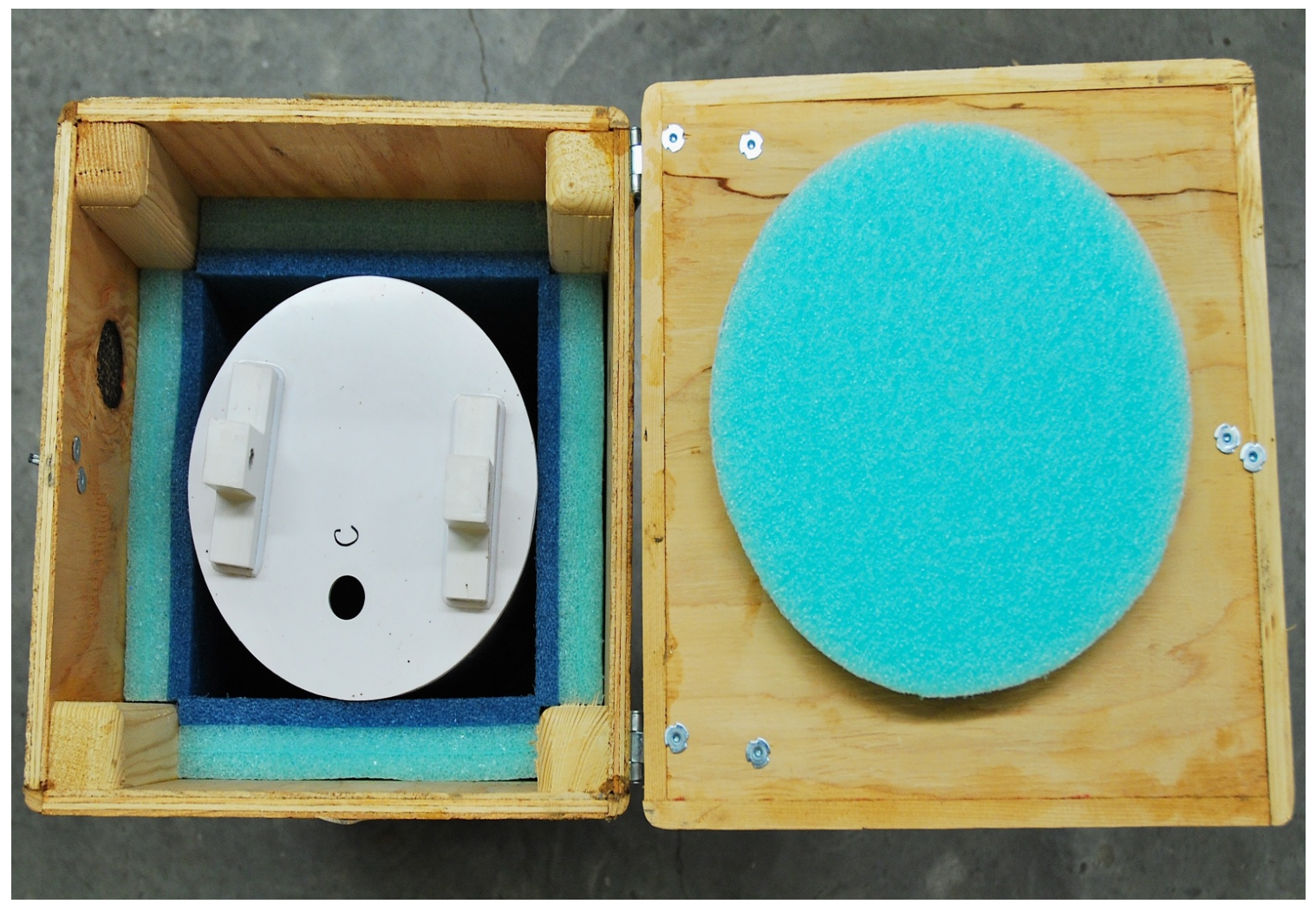


Figure 6: Electronics Package

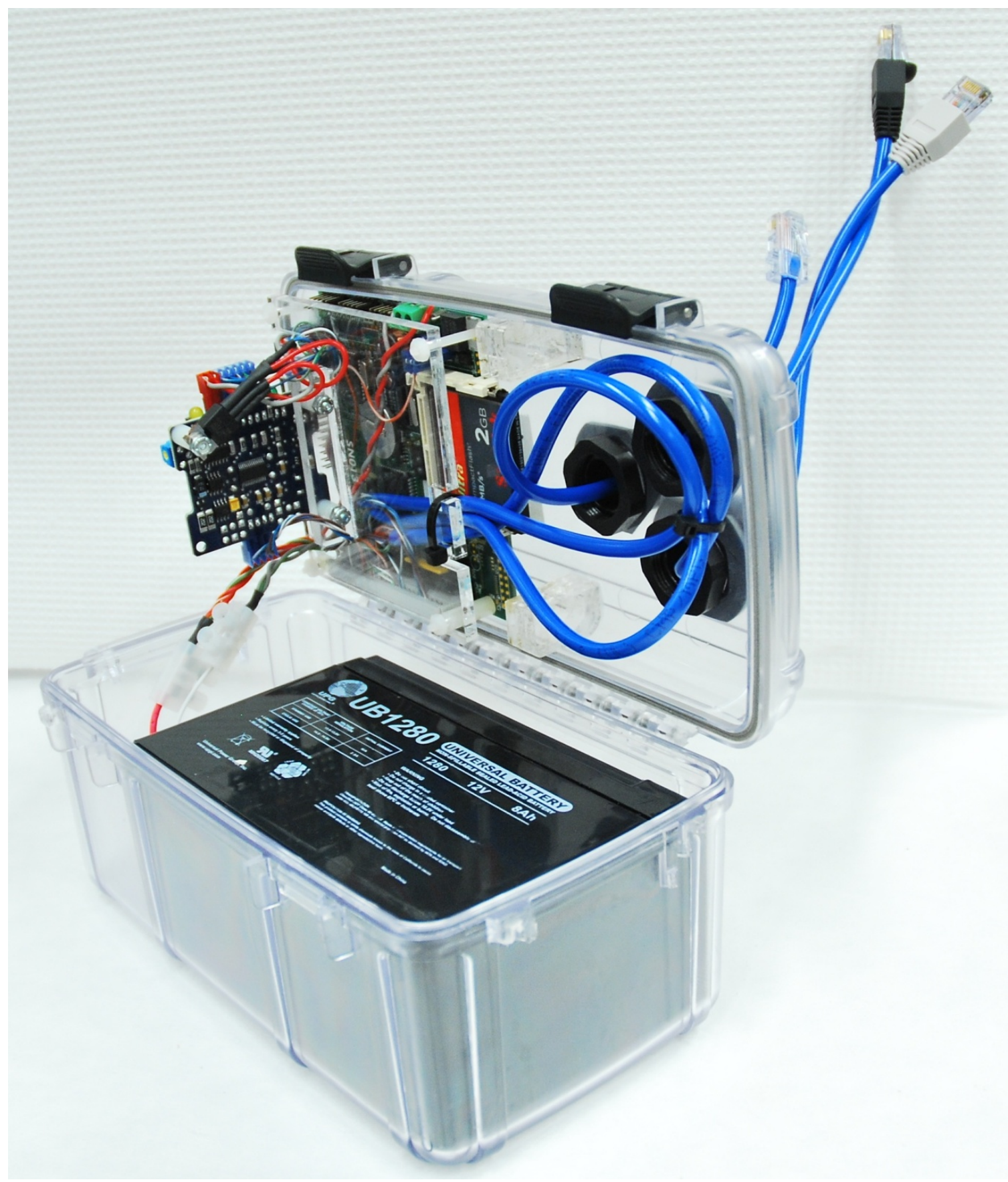


Figure 7: Battery

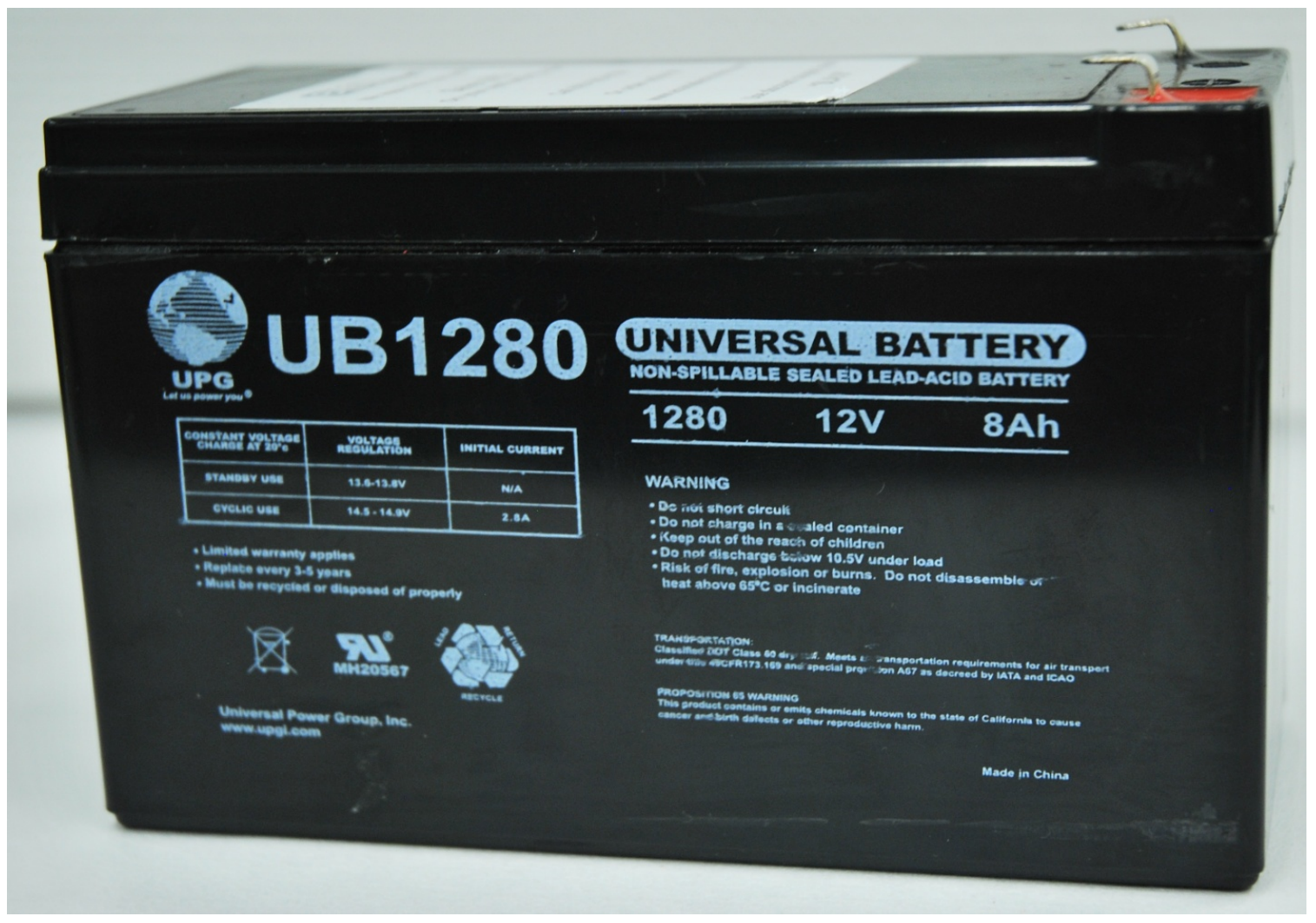


Figure 8: Peristaltic Pump

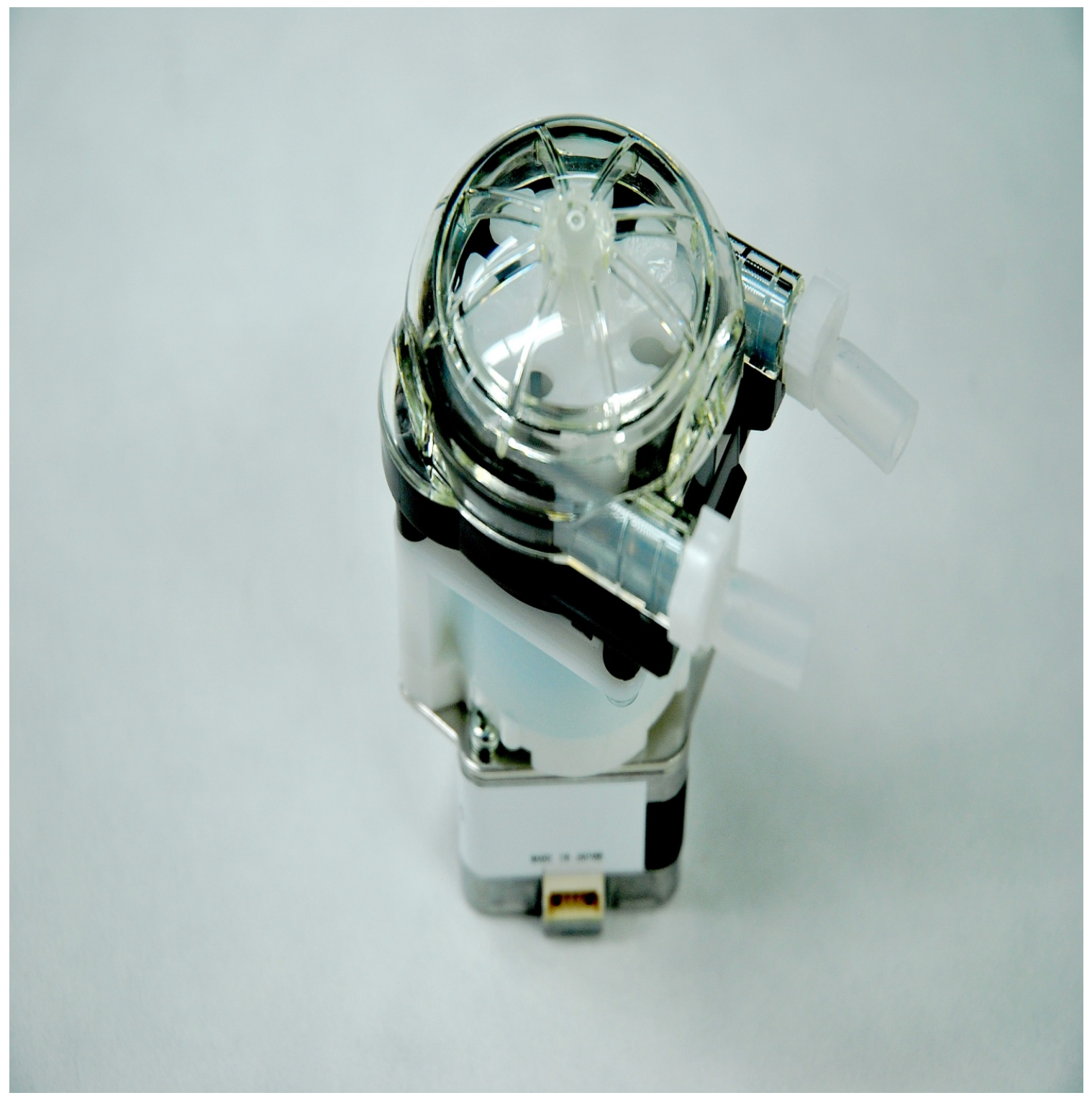


Figure 9: Solenoid Pinch Valve

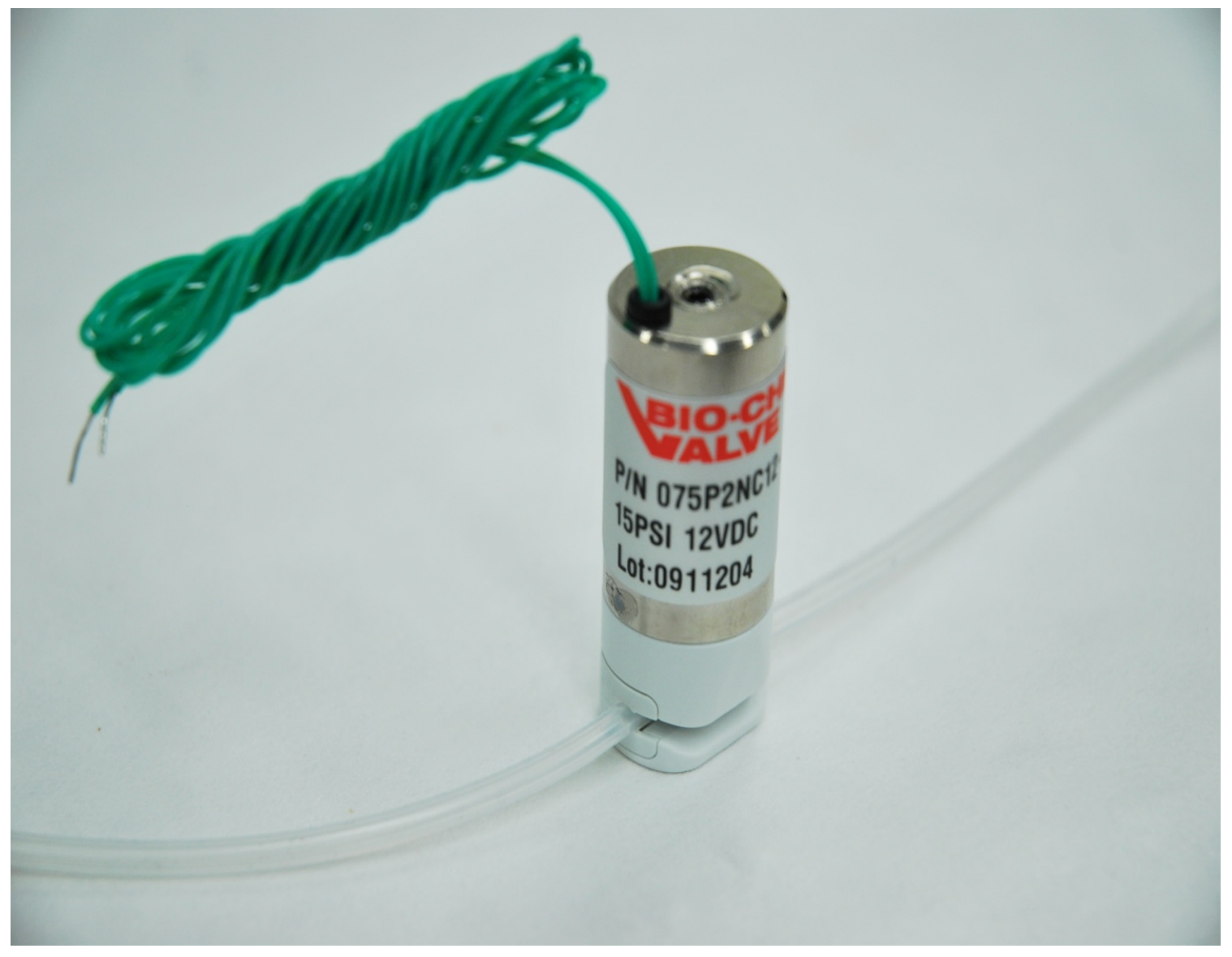


Figure 10: Overflow Reservoir

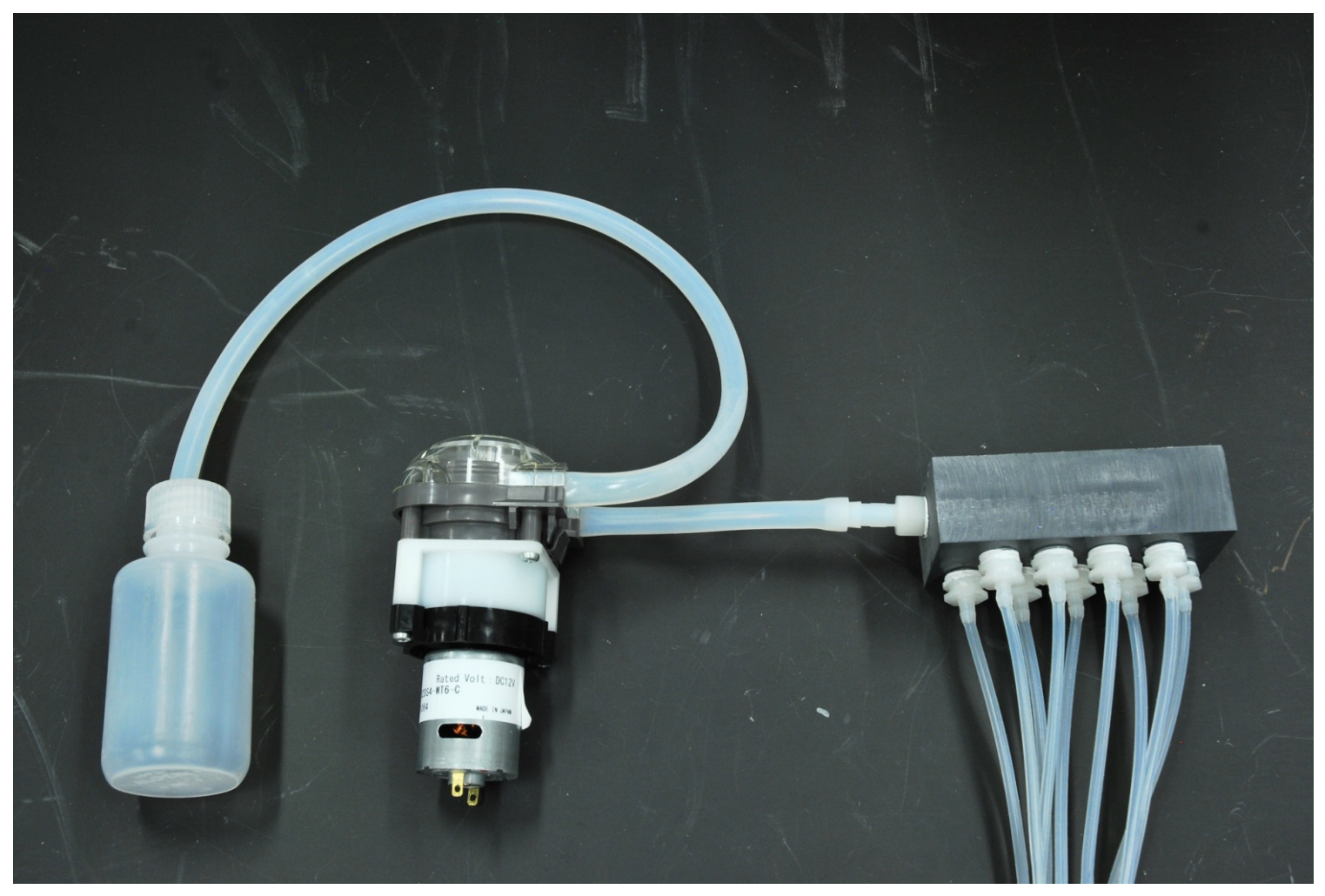


Figure 11: O-Ring Seal

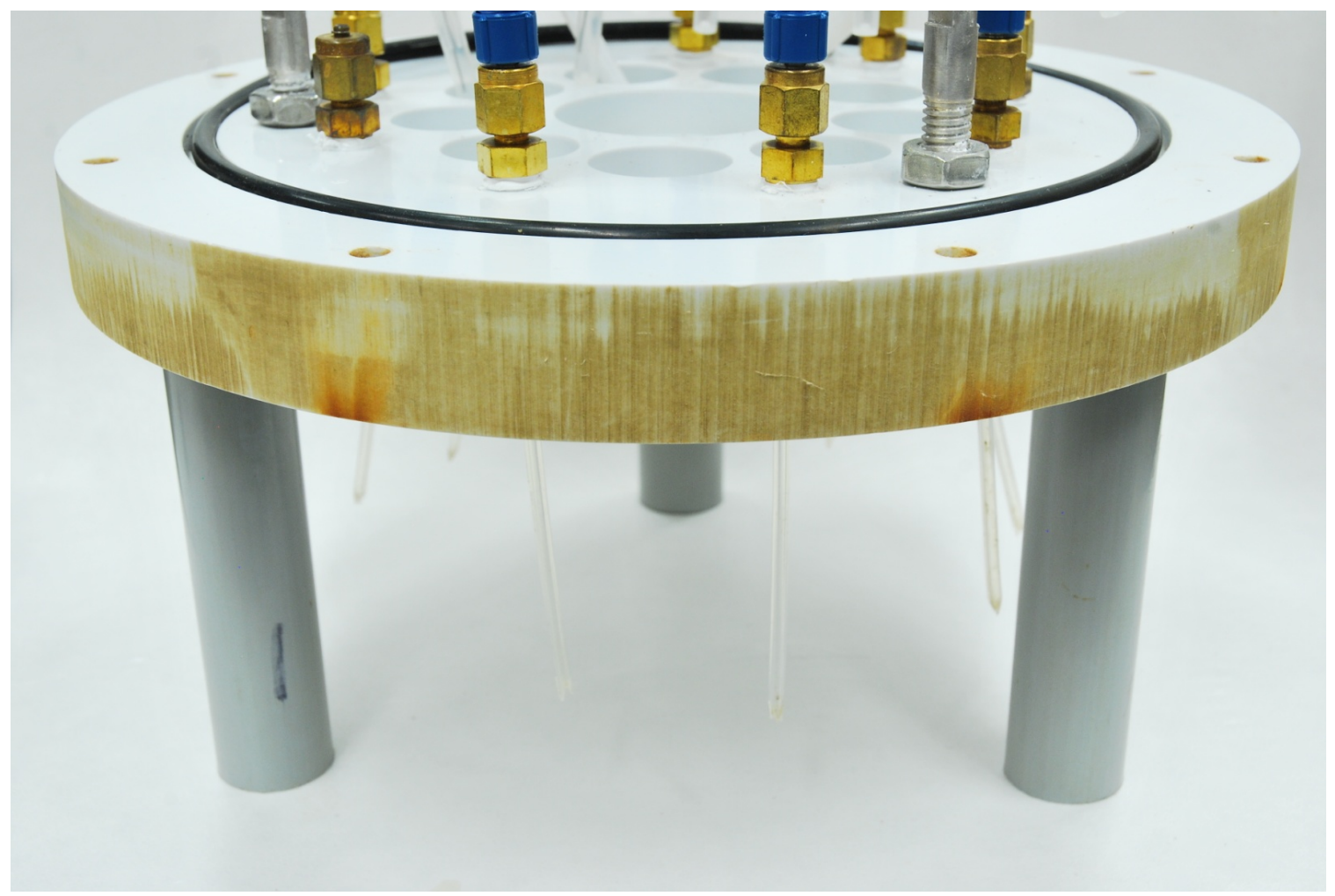


Figure 12: Waterproof Cable Gland

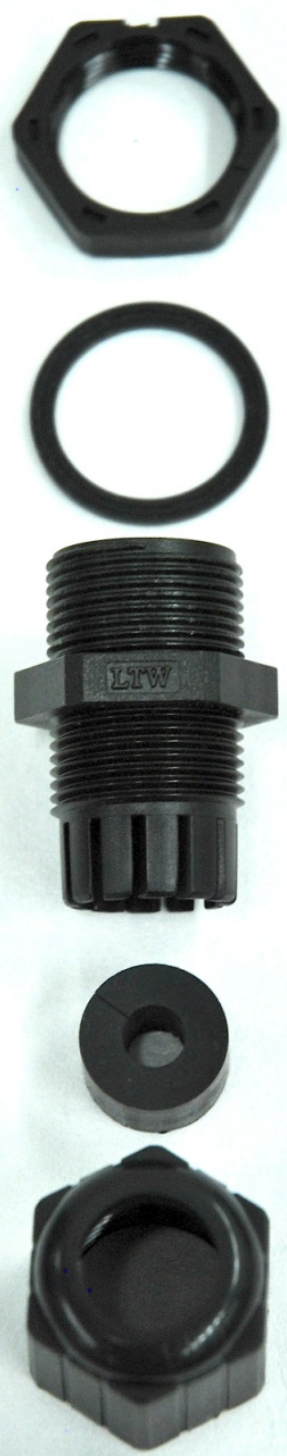


Figure 13: Remote Unit

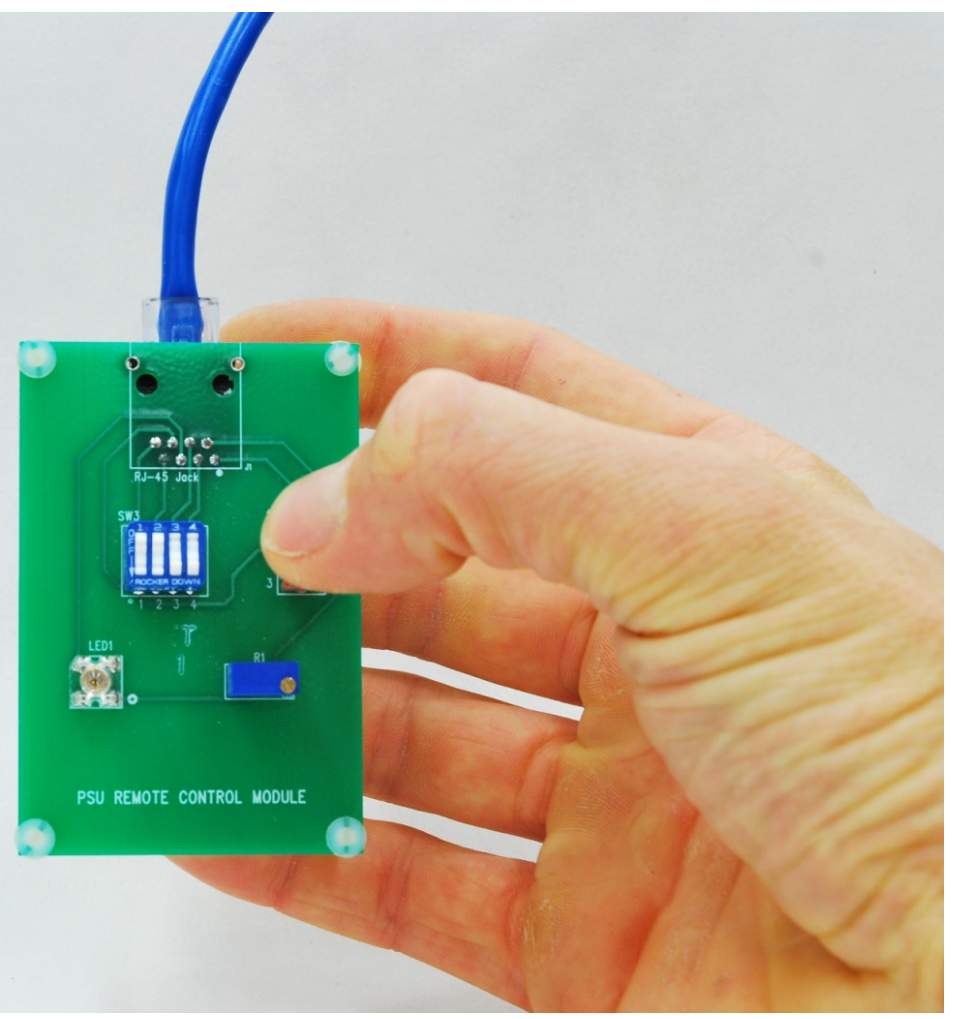


Figure 14: Simplified Flowpath for the Daily Composite

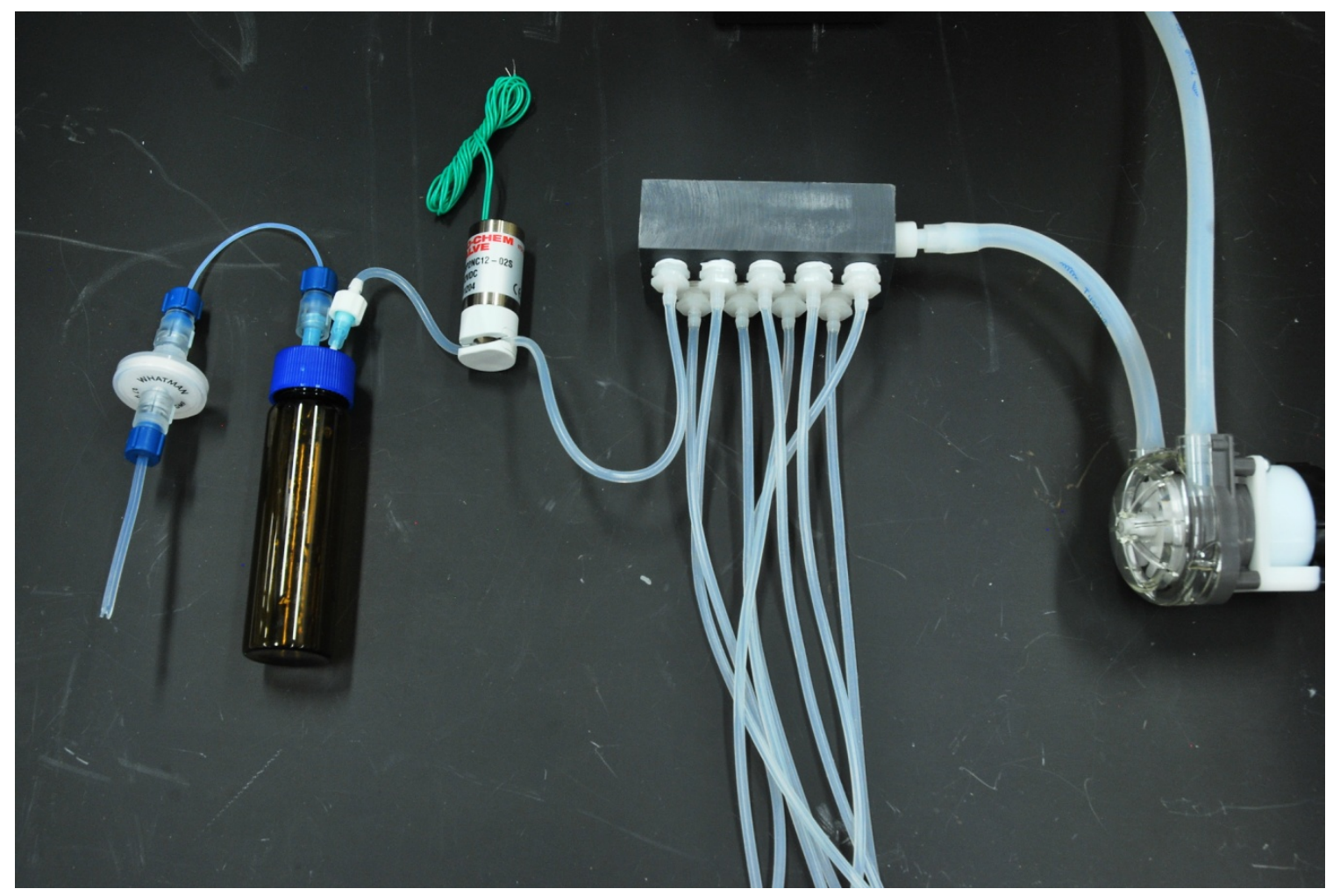


Figure 15: Simplified Flowpath for the Weekly Composite

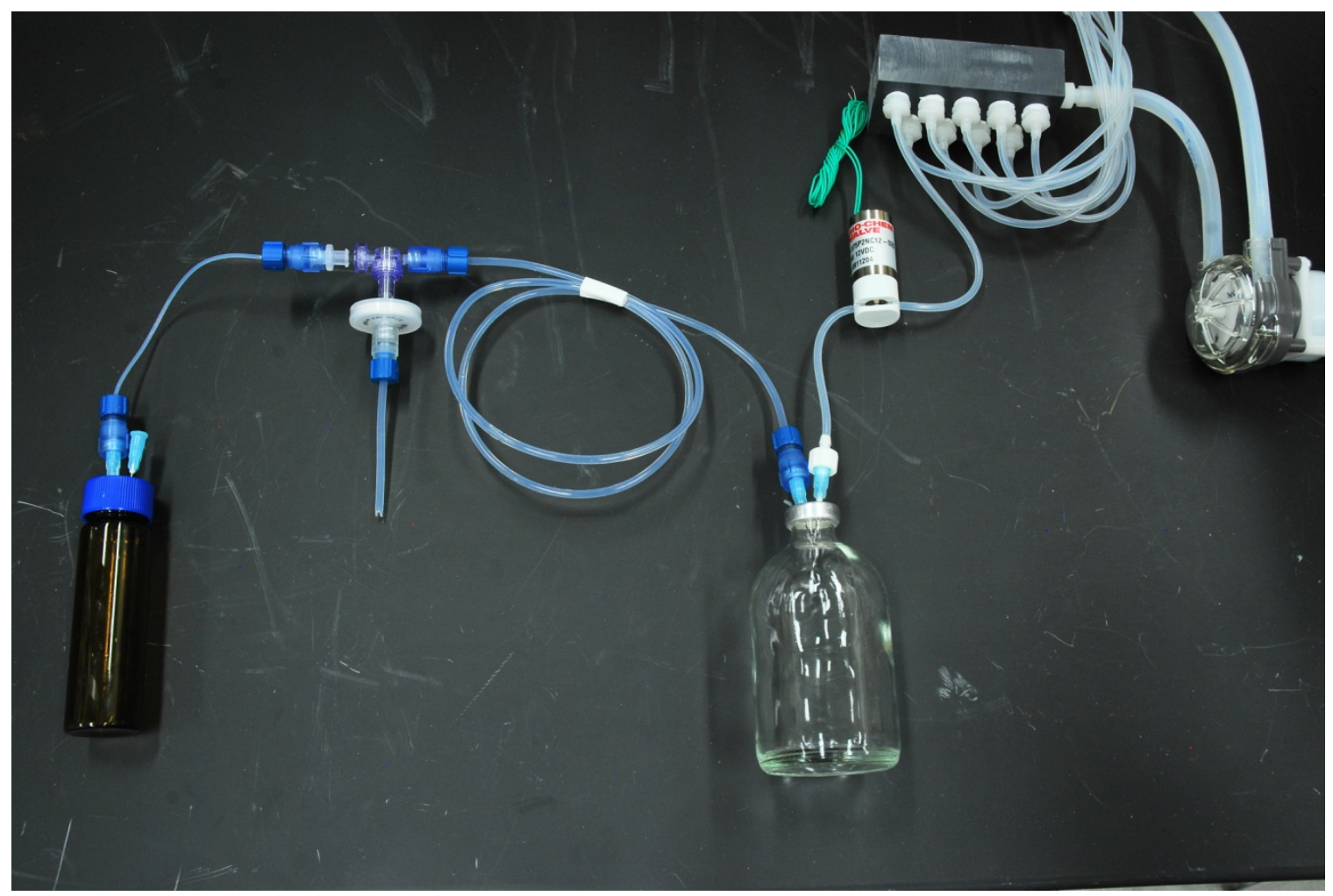


Figure 16: SVAWS During Deployment

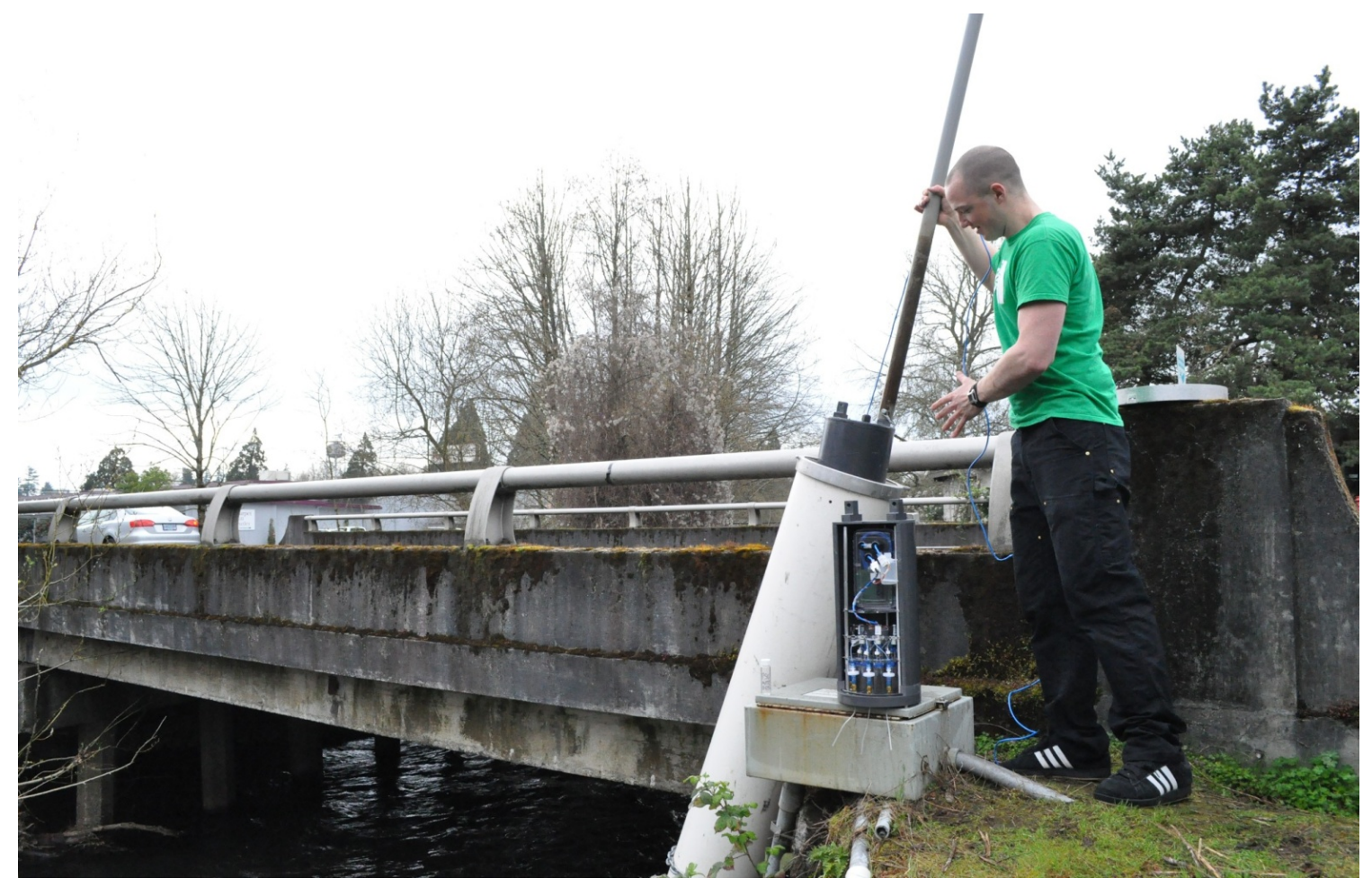


Figure 17: Securing the Field Installation

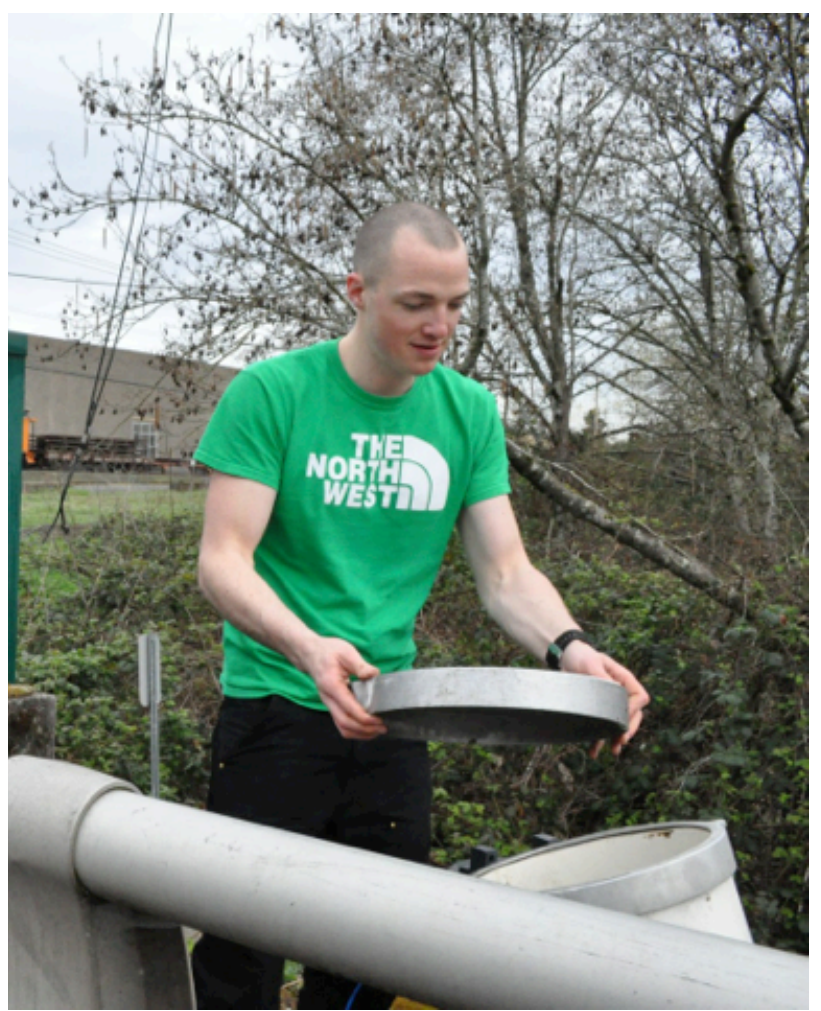




\section{REFERENCES}

Analytical columns. (2013). Volatile organic analysis (VOA). Retrieved from: http://www.analyticalcolumns.com/Vials/voavials.html, pp. 1-2.

EPA. (2010). The National Rivers and Streams Assessment (NRSA) 2008-2009 Draft Report. Washington, DC: Environmental Protection Agency (EPA). Retrieved from: http://water.epa.gov/type/rsl/monitoring/riverssurvey/

Grube, A., Donaldson, D., Kiely, T., \& Wu, L. (2011). Pesticides industry sales and usage: 2006 and 2007 market estimates. Washington, DC: U.S. Environmental Protection Agency (EPA). Retrieved from: http://www.epa.gov/opp00001/pestsales/

Hildebrandt, A., Lacorte, S., \& Barcelo, D. (2006). Sampling of water, soil and sediment to trace organize pollutants at a river-basins scale. Analytical \& Bioanalytical Chemistry, 386(4), pp. 1075-1088.

Korfmacher, J.L., \& Musselman, R.C. (2007). Evaluation of storage and filtration protocols for Alpine/Subalpine lake water quality. Environmental Monitoring \& Assessment, 131(1-3), pp. 107-116.

McCall, W. (2005). Evaluation of a small mechanical and pneumatic bladder pump. Ground Water Monitoring \& Remediation, 25(2), pp. 142-153.

Stephenson, J.B. (2004). Watershed management: Better coordination of data collection efforts needed to support key decisions. GAO Reports, pp. 1-155.

USGS National water use data system: An optimal data base? (1984). H.M. Review of Public Data, 12(2), pp. 97-105.

USGS. (2010). National Field Manual for the Collection of Water-Quality Data. Washington, DC: USGS. Retrieved from: 
http://water.usgs.gov/owq/FieldManual/chapter4/html/Ch4_contents.html

Water quality: Key EPA and state decisions limited by inconsistent and incomplete data. (2000). GAO Reports, pp. 1-78. 


\section{APPENDICES}

\section{Appendix A}

\section{USGS Tester Training Information}

\section{SVAWS OPERATION INSTRUCTIONS}

TO BEGIN SAMPLING NORMALLY:

1. Connect the remote and lower the sampler so the sampler bottom touches the water surface

2. Code in 0010 to the remote (Figure 1)

3. Press and hold the remote pushbutton switch. The remote LED will flash shortshort-long-short if you keyed in the code properly.

4. When the LED stays lit, pause $\sim 1$ second then release the pushbutton.

5. In 10 seconds, the remote LED will flicker for $\sim 5$ seconds indicating the field initialization was successful. At this point the pump is pressurizing the sample vials so the bottom of the sampler should be submerged

6. Disconnect the remote, cap the cable end and let the sampler float in the field installation

7. The sampler is initialized. Sampling will begin at noon and end at 6am in 7 days

TO BEGIN LATE SAMPLING:

If one arrives to the field after noon but before $6 \mathrm{pm}$ :

- Preform all the steps outlined for a normal sample but replace the remote code with 0011 (Figure 2)

- The sampler will skip sample 1 aliquot 1 and begin with sample 1 aliquot 2 at $6 \mathrm{pm}$.

- The weekly composite will not be affected as its aliquots are taken following aliquots 2 and 4 of each daily composite

TO RESET THE SAMPLER:

- Key in 0001 to the remote (Figure 3)

- Press and hold the remote pushbutton

- 1 second after the remote LED stays lit, release the pushbutton

- The sampler is now shut down indefinitely
NOTE: $A$ " 1 " on the remote is achieved by depressing the bottom side of the switch so that the top of the switch (under the switch number) protrudes from the switch housing

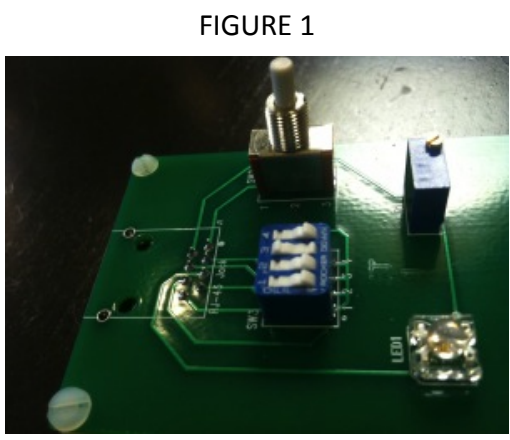

FIGURE 2

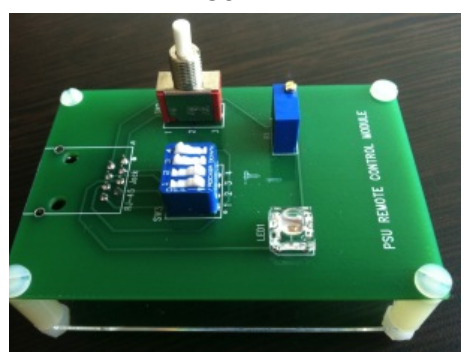

FIGURE 3

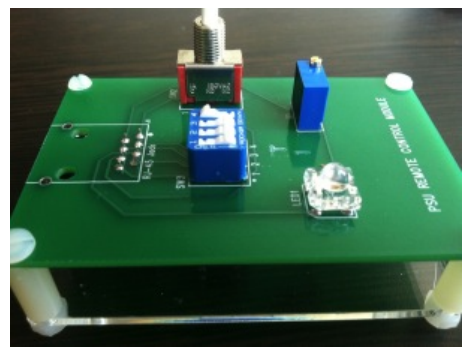




\section{DEBUGGING CODES}

TO TEST THE VALVES AND PUMP:

- Key in remote code 0100 (Figure 4)

- Press and hold the remote push button switch

- The remote LED will flash then the valves will open sequentially and the pump will run in forward and reverse

TO TEST THE MANIFOLD FOR LEAKS:

- Connect a vacuum gauge to the manifold

- Input code 0101 to the remote (Figure 5)

- Press and hold the pushbutton until the pump shuts off ( 30 seconds)

- Monitor the manifold's pressure to determine if leaks are present

FIGURE 4

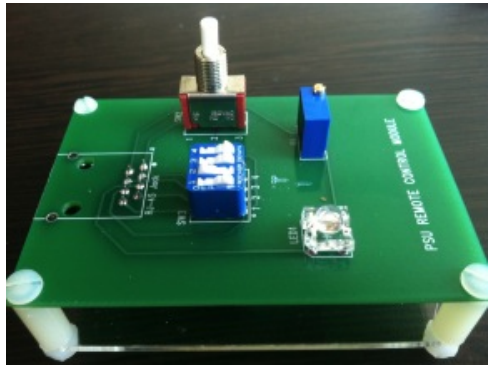

FIGURE 5

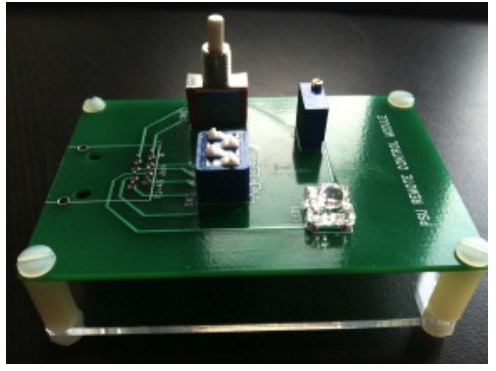




\section{Appendix B}

\section{Participant Survey Request}

From: Nicholas Fitzgerald

To: USGS SVAWS Testing Members

Re: SVAWS Survey

Date: August 6, 2013

Esteemed professionals,

Below is a link for a survey related to the performance of the SVAWS (aka the "Pankow") water sampler. At your earliest convenience, please complete the survey by following the link. Further, if there is someone in your office that has moderate experience with the SVAWS and is not copied above, please forward this email to them. Your help is greatly appreciated.

https://qtrial.qualtrics.com/SE/?SID=SV_dja70Hio7eC0Dtz

Sincerely,

Nicholas Fitzgerald

Nicholas Fitzgerald, BS Environmental Engineering, MS Candidate 


\section{Appendix C}

\section{Statistical Results of Likert Scale Survey}

1. Please enter your name and the field office you work for:

\begin{tabular}{|c|c|c|c|c|c|c|}
\hline \multicolumn{7}{|c|}{ Shannon Stubbs, Iowa WSC } \\
\hline \multicolumn{7}{|c|}{ Jeff Frey } \\
\hline \multicolumn{7}{|c|}{ Chevelle Schreiner, Nebraska Water Science Center } \\
\hline \multicolumn{7}{|c|}{ Denis Scanlan Wisconsin Water Science Center } \\
\hline \multicolumn{7}{|c|}{ Teresa Rasmussen, Kansas Water Science Center (serviced a Missouri sampler) } \\
\hline \multicolumn{7}{|c|}{ Megan Shoda, Indiana Water Science Center } \\
\hline \multicolumn{7}{|c|}{ 2. How difficult was operation of the SVAWS sampler? } \\
\hline \# & Answer & Min Value & Max Value & $\begin{array}{c}\text { Average } \\
\text { Value }\end{array}$ & $\begin{array}{l}\text { Standard } \\
\text { Deviation }\end{array}$ & Responses \\
\hline 1 & 1 & 30.00 & 100.00 & 63.57 & 23.22 & 7 \\
\hline
\end{tabular}

3. How difficult was maintenance of the SVAWS sampler?

\begin{tabular}{|c|c|c|c|c|c|c|}
\hline$\#$ & Answer & Min Value & Max Value & $\begin{array}{c}\text { Average } \\
\text { Value }\end{array}$ & $\begin{array}{c}\text { Standard } \\
\text { Deviation }\end{array}$ & Responses \\
\hline 1 & 1 & 9.00 & 92.00 & 51.29 & 29.26 & 7 \\
\hline
\end{tabular}

4. Based on your experience, do you believe the SVAWS sampler is more or less costly to install and operate than other automated samplers (such as those made by Teledyne Isco)?

\begin{tabular}{|c|c|c|c|c|c|c|}
\hline$\#$ & Answer & Min Value & Max Value & $\begin{array}{c}\text { Average } \\
\text { Value }\end{array}$ & $\begin{array}{c}\text { Standard } \\
\text { Deviation }\end{array}$ & Responses \\
\hline 1 & 1 & 9.00 & 100.00 & 59.83 & 31.31 & 6 \\
\hline
\end{tabular}

5. Does the design of the field installation and SVAWS sampler allow for installation at many or few field sites? IE are many or few sites available for sampling

\begin{tabular}{|c|c|c|c|c|c|c|}
\hline$\#$ & Answer & Min Value & Max Value & $\begin{array}{c}\text { Average } \\
\text { Value }\end{array}$ & $\begin{array}{c}\text { Standard } \\
\text { Deviation }\end{array}$ & Responses \\
\hline 1 & 1 & 10.00 & 100.00 & 49.00 & 34.60 & 7 \\
\hline
\end{tabular}

6. How many times did you note that a fully charged battery onboard the SVAWS discharged completely during a one 
week field deployment?

\begin{tabular}{|c|c|c|c|c|c|c|}
\hline$\#$ & Answer & Min Value & Max Value & $\begin{array}{c}\text { Average } \\
\text { Value }\end{array}$ & $\begin{array}{c}\text { Standard } \\
\text { Deviation }\end{array}$ & Responses \\
\hline 1 & 1 & 0.00 & 0.00 & 0.00 & 0.00 & 4 \\
\hline
\end{tabular}

7. Do you believe the SVAWS sampler has the potential to serve as a platform and be used for various future applications?

\begin{tabular}{|c|c|c|c|c|c|c|}
\hline$\#$ & Answer & Min Value & Max Value & $\begin{array}{c}\text { Average } \\
\text { Value }\end{array}$ & $\begin{array}{c}\text { Standard } \\
\text { Deviation }\end{array}$ & Responses \\
\hline 1 & 1 & 49.00 & 100.00 & 73.43 & 21.54 & 7 \\
\hline
\end{tabular}




\section{Appendix D}

\section{Essay Responses to Survey Questions}

\section{What do you think would simplify the operating procedure of the SVAWS sampler?}

I never had the training nor actually worked on operation of maintenance so I would leave this to Megan, Denis, Shannon, and others who did this.

The sampler did not come with detailed instructions. There were many small parts and if one part were to be installed incorrectly, the whole processes would be affected and the sample void. If one were to discover a missing part upon checking the sampler for deployment, the whole process had to be reversed. The acrylic plate had to be removed but not until all of the needled were first removed.

I can't think of anything that would simplify the operating procedure of the sampler. It is fair easy and straight forward with regards to the operating procedure. One item that has effected the operation of the samplers in the Wisconsin district are the small (fins/protrusions) on the outside of the sampler. When high water inundated the sites and caused the samplers to rise in the PVC tubes, the samplers would occasionally hang up in the bored out PVC tube holes and remain there out of the water after the water receded. No samples were collected when this happened. Maybe it was a function of the PVC tube holes being to big. I'm not sure.

The biggest operational fear was that we might return to the site a week after deployment and find that the sampler didn't do anything. Would be nice to have a more convincing indication than blinking lights that the sampler is working when it's deployed. Add the ability to hook the sampler up to a tablet or laptop to deploy it and verify that it's working. This would make the switch a little more sophisticated and useful. It was very inconvenient when our procedure changed midway through the study so that we loaded the spike vial at the site after collecting a sample vial to add spike solution to. Although this wasn't a design flaw in the sampler, it did affect the operating procedure in a negative way. A reminder that lab procedures affect operation efficiency.

The actual operating procedure is pretty easy - take the sampler out of the box, put it in the PVC tube, press start command. If there was another way to open and close it, that would save time. Could we do watertight closures another way? Hinges that snap in place? The nuts and bolts get a little tedious and require tools - another thing to remember to bring along on your trip.

An easier to use digital controller with builtin commands, such as clean cylce, begin weekly sampling, etc. The current method is ok, but it seems like it could be simplified so that you don't have to hand someone a list of codes to operate it. 
Having the sampler be cordless would be fantastic. The ethernet cable posed multiple problems in Iowa (wasn't long enough so we had to put our own on, too many connections to check when it wouldn't operate, and when raising and lowering the sampler, the possibility of it slipping existed - which meant the ethernet cable was sometimes bearing a heavy load unexpectedly). Also, the lip at the bottom of the cover causes issues when taking the cover off because wires, tubing, shelves, etc get caught on it. Very frustrating when you're working under a deadline and in the sun on a bridge.

We need to figure out what causes low volumes of water. Not sure if there is some issue with Midwest streams compared to Oregon streams but we seemed to have

After cleansing the system with soap, tap water, and DI, we were to run methanol through each sample tubing. In order to do this without wasting copious amount of methanol, we had to connect the sample needle of each sample tube individually into the waste bottle and use a small beaker with methanol which became very time consuming.

I think more options with the remote to troubleshoot individual sample lines would help. Maybe there could be a different way to test each sample line's pressure. Another way to simplify maintenance is to have more user friendly parts on the sampler. I had an issue with the Ethernet cable on one of the housings and could not replace it easily. The outer sheathing had been stripped off of the cable due to the constant movement of the sampler of the tube. This caused the sampler to not be able to communicate with the remote. I tried to replace the cable but was unable to get the cable through the water proof fitting. Try and make all parts serviceable by field technicians in the field. Another idea is to encapsulate all electronics in water proof boxes so that if there is a leak in the housing there will not be any corrosion on the electronic connections.

It would be nice to have a diagnostic procedure that cycles through each vial and valve, actually pulling water into each vial, to determine whether the system was functioning properly. Like the entire week's sampling procedure condensed into less than one day. The test code that checked the pump and valves was helpful, but didn't indicate whether adequate vacuum was occurring to pull water into each vial. One of our samplers repeatedly had trouble filling vials 5 and 6, but we didn't realize that until end of the week, and then still didn't know whether it was a system problem or blockage or what. We would have recognized it early with a more complete diagnostic test in the lab prior to deployment.

I had a lot of little things that didn't work that I was always trying to figure out. The weekly composite was hit or miss and I spent a lot of time trying to figure out why or why certain vials didn't sample for a given week. The instrument seemed fragile - like it was easy to knock a connection loose. Putting the housing on and off requires two people and you feel like you are forcing it to go down over tubes and connections. It was not a smooth fit, I think maybe because of the weekly composite loop and the tension you have to keep on the ethernet cable.

A more detailed manual with step by step instructions would be of great help. This should include a troubleshooting section documenting common problems and suggested corrections. A more thorough training would be helpful as well. This should detail expected performance as well. One issue we had involved overfilling of the vials. We did not know we had an issue until later down the line because we did not know how much water volume to expect. 


\section{What changes to the SVAWS sampler would lower operating costs?}

The sampler should be able to be lifted, cleaned, and prepared by one technician. It always took 2 people to balance it and lift it due to its awkward weight distribution. It also took about an hour to clean the sampler and another 30 to 45 minutes to ready it for deployment. This usually required 2 technicians and costing more than if the sampler could be handled by one technician.

Try to eliminate some of the fittings associated with each sample line. Too much time is spent having to check and recheck each and every sample line to insure that there is no pressure leak in any of the lines. The cost, time and maintenance headaches would be lower if the system had less connectable parts.

Actual operating costs (supplies etc) seem pretty low. Best thing is to simplify cleaning and prep to reduce required maintenance time.

Find a better way to seal the container that it comes in. I think most of the operating costs are due to equipment getting ruined due to immersion. I wonder if there is a way to make it so the entire bottom section is one piece so and then attach a top piece so that only under extreme cirumstances would there be water around areas that have connecting pieces, which are going to be more susceptible to leaking. 


\section{What changes would reduce costs of the SVAWS field installation?}

The large piece of PVC that was needed to house the sampler was very costly. At our site the piece needed to be very long and therefore was slightly unstable. Brackets were needed to hold it against the pier which required more special equipment and a crew of three technicians to install. Also, we did not have enough time to obtain proper permission to install the housing on the bridge.

I don't have any suggestions on how to reduce costs for the field installation of the samplers. It is pretty easy to install the PVC tubing and the sampler.

Field installation was relatively inexpensive. Most difficult aspect of installation was maneuvering the long 12" PVC tube.

See below. Field installation is costly due to materials cost and the number of people needed to install. At my Zionsville site, we had $30 \mathrm{ft}$. of pipe, weighing about 300 pounds. It took 4 people to install vertically off the bridge.

If it could be made to sit afloat on its own it would not require such an elaborate (in some cases) setup. This is especially true in cases where you have tall bridges and steap banks. Costs (supplies and time) for those could be substantial. 
12. What changes could be made to the SVAWS system so that more field sites could be accessed?

The SVAWS was not conducive to Nebraska's mud-bottom and high sediment level streams. The sample tubing would often be obstructed, not allowing a proper sample to be collected.

I can't think of any changes to make more field sites accessible. The installation is easy and the samplers are self contained units so they should be fairly easy to install and use anywhere.

Mount the sampler on the bridge deck and pump water up to a basin from which sample is drawn, rather than deploying sampler directly in the stream.

Site selection and installation were problematic because of the vertical deployment of the sampler and the cost and installation issues with having long lengths of 12" PVC pipe. The pipe is expensive and heavy, and requires a trailer to load and transport. Unless there is a bridge pier to attach to, the PVC can't be installed, and even then it has to be somewhat protected. I think the sampler I lost was due to the rough rise and fall of water (or debris jams?) when flow went up and then fell at my site. How can this be used without the PVC pipe? How can this be deployed at a site with low, gradual banks and no bridge? Maybe a bank anchored standpipe sort of set up would work?

It would need to be more bouyant, because for most of our streams stream depth (too shallow) was the limiting factor. 
13. What other studies can you envision preforming with the SVAWS system?

I'd like to see it used with NO3 sampling.

The idea of the sampler is a great one but I would not recommend using the sampler for any other studies at this time.

Just about any study that can use $40 \mathrm{ml}$ or less of sample volume for the testing. Obviously that will be the limiting factor with the sampler. If a particular parameter needs more then the sample vials can hold to run the tests then the SVAWS sampler will not work.

If the data are reliable, it could be useful for studying other organic contaminants such as wastewater compounds and pharmaceuticals. This would be interesting in urban areas looking at fate and transport during runoff and in wastewater discharges. But I have concerns about sample holding times.

Daily composites would lend themselves to a small scale, intensive watershed study. 


\section{What would you change about the SVAWS system?}

Better sharing of data (volumes of sample, which bottles aren't filling, etc..) between users so we can learn what may be going wrong in the field. However, with these intensive sampling schedules with MSQA and SESQA it makes it tough to communicate amongst groups.

Due to the large quantity of small parts there are many places that air can be let into the system. If any of the couplers or tubes were to come loose the entire sample is affected. Also, after a sample is finished it sits in the vial for a week. The inside of the sampler was at a much higher temperature than the water. I imagine that being in a warmer temperature for that long could affect the sample. I do not have any proposed solutions to this, only an observation of an opportunity for sample to be stressed. The computer aspect of the sampler, or the "brain" as we referred to it, was a little complicated as well. The remote did not come with instructions or codes. It was sometime unclear which way was on or off for each switch. I wish there would have been a better indicator that the system had been activated and was working properly. For an electrical engineer this may have seemed elementary but the personnel using the system were not trained in that field. So I would suggest a more "user-friendly" remote.

I would encase all electronics in water proof boxes. Have only parts that will not be adversely affected by contact with water outside of the boxes. I would try to remove as many moving parts as possible so that there is less of a chance of losing pressure throughout the system. There are too many connections that can pose a problem which makes it very difficult to troubleshoot any issues, especially in the field. Make the Ethernet cable either a higher quality grade cable to prevent stripping of the sheathing when the sampler rises and falls in the PVC tube or use something different. Make it easier to service (swap out) the cable if there is a problem. Make the remotes much more durable for field work. I would also figure out a way to easily troubleshoot the problems with the weekly composite samples. I followed all of the suggestions for the weekly samples but I had issues with $95 \%$ of the samples (mainly a lack of samples). Don't know why the samples were not collected but it frustrated me to no end trying to get the samplers to collect the weekly composite samples.

Permanently attach the legs to the bottom of the sampler. This was an unnecessary source of frustration because it wasn't easily fixed and if the legs popped off when the sampler was lowered into the tube there's no way of knowing it and the entire week's samples fail because the inlet tubes are all smashed under the weight of the sampler.

I would like to see the sampler equipped with a command to sample immediately in each vial (maybe one at a time or the user can choose which line to sample from). If I had problems with a sampling line (I get the sampler back from the field and there is no sample in one vial) I did a couple things to try and troubleshoot the issue. I would replace tubing, tighten connections, etc. but then I was never really sure if I solved the problem or not because I couldn't test the new parts. If I could test the sampler right away and run DI water through the lines, I would have more confidence with my next deployment. I also had to replace all the ethernet cables on my samplers because the ones supplied were not long enough. It would probably save the lab and the water science center some time if we talked about what length cable we needed. Also, I had a cable severed in the field and one was damaged in shipping (the casing was cut). Maybe we can protect the cables?

I have included some comments in the above sections that relate to this but in addion to those I comments I would recommend a better "foot" design. Rather than having feet that can fall off we thought that a perforated extension of the container would work better. It would allow water to pass through while providing support for the entire circumference. The feet easily fell off and would not keep the sampler standing if they were to come to rest on an uneven area of the stream channel bottom. Lastly, if this is used during high runoff events the filters clog quickly before there is enough water for a sample. A larger filter would allow for sampling in this conditions. 


\section{What did you like about the SVAWS system?}

The idea of it. I think it's a really great idea and I really want to see it become a viable sampling option.

I liked having backup systems so they could be maintained in the office and then exchanged in the field.

I do like that the sampler takes small samples. Other automatic samplers collect much more water than needed.

I like the portability of the system and the relatively easy installation of the sampler.

I like cleaning in the lab/office and swapping out complete samplers during each field visit. I like the simplicity of the field procedure...easy to hook up, easy to turn on, and all done (if everything worked right). Best thing is the small sample containers and the compact size of the sampler.

When things were working, I liked using the sampler. I felt rewarded with full vials and confident that the sampler was working by collecting composites appropriately.

I was a little skeptical at first but I was impressed overall with how consistently the system worked. 


\section{What didn't you like about the SVAWS system?}

The never knowing if I was going to have a sample Monday afternoon when I went to pick it up. I also really did not like the inlet tubing. Iowa river bottoms are muddy. We had a few times where we would possibly have gotten a sample, but didn't because the tubing was impacted.

Need better training and more time to get staff familiar with all aspects of the system. Once we got the crimping tool things improved but

The system was constantly breaking. Either a leg would fall off, a plate would break, or the vials would overfill. It was also very top heavy and required two people to remove it from the box, clean it and prepare it for deployment. The project that we used the sampler for was very intense and the sampler really slowed us down and cost a lot of money. Most of the weight at the top of the sampler was due to the battery. Does the sampler need such a large power source? Would a smaller battery suffice?

I didn't like the inconsistent sample volumes collected by the sampler. I followed all the procedures, that were demonstrated by Wentai, from the very first day but the sampler collected inconsistent sample volumes throughout the project. I didn't like the fact that there were way to many points in the sampler that could fail. There were too many parts associated with the sample lines. I also didn't like the fragile nature of the sampler. The samplers are subject to large rises and falls in the stage of the sites and they seem to be very fragile in how they are built. The system needs to be made much more rugged to with stand the punishment that they take in very flashy water.

Lots of fittings and small tubing, easily blocked by sediment and debris and easily knocked loose during cleaning and prep for next deployment. Hard to tell if something was caught or knocked loose when lowering the cover down over the sampler.

I'd like to see a better shipping technique. I had samplers arrive with broken feet and broken acrylic plates. It's tough to start with something new when it's already broken.

There were a lot of small pieces. Small pieces easily are lost and because there are so many it can be difficult to put everything back together once it is apart. 
17. If your SVAWS ever flooded, what was the cause of the flooding? Do you have any comments related to the SVAWS flooding?

\section{NA}

\section{See Megan's response}

Our samplers were never "flooded". There would, however, occasionally be water in the sampler due to the overfilling of the vials.

One of the SVAWS was flooded due to the fact that one of the fittings on a sample line came off due to the pressure buildup and this allowed a direct, unrestricted line for the water to flow from outside of the sampler into the inner cavity of the sampler. I believe that if some of the connections associated with each sample line were eliminated then there would be less chance of the flooding issue to occur. Once again too many parts associated with each line causes too many links within each line that can fail.

Our sampler did flood once. Really don't know why. There was a large runoff event during the week it flooded, so I'm guessing it was related to that. Maybe the sampler didn't rise and fall smoothly in the deployment tube. Maybe it got hung up on debris inside the tube. Maybe water leaked through the bottom seal, but I don't think so because we were pretty careful about checking the seal.

I had a sampler flood and I think the cause was leaky connections between the inlet tubes. Wentai told me the inlet tubes expand and shrink over time and the connections come loose. Water never made it to the filter in my case. I had no way to tell how often I should be replacing parts due to this kind of thing, or actually how often I should replace any part. If I had no volume in a vial, I usually just replaced all the parts in that line but I never knew what the cause was.

Not a good seal of the container, see comments in above question: "What changes to the SVAWS sampler would lower operating costs?" 


\section{Final Comments?}

Overall, I loved the Pankow. I was the only one who really did anything with it in Iowa. Other than a single week that it was deployed, I was the one who went and serviced it every time, so I think I know it pretty well. My chief complaint with it is the sheer number of connections - tubing and electrical - that it has. There are just too many places for it to fail. It was really frustrating to deploy it and then not have the remote work. Or deploy it and have the remote work and come back a week later to no samples - and no real idea why. The times that happened I chalked it up to a loose fitting in the tubing, but I'm really not sure what the deal was. I would definitely be interested in knowing how the product is changed and also in working with it again in the future.

Honestly guys, I had very little to do with any of the operation and maintenance. I look forward to hearing from Megan and other field staff who did the work.

There were very few times that the SVAWS ever produced a viable sample for each day and the composite. Sometimes it was operator error, but mostly I feel that the sampler was not ready for use with the USGS. I really wish there would have been more field testing. The system was costly, finicky, and complicated. The site that we had originally planned to use the sampler had to be changed due to the specific deployment procedures for the sample. To access the sampler one technician would have to climb a ladder that leaned against the pier and was not very stable due the the nature of the bank. The other technician would have to stand on the bridge and catch the rope by a carabiner that was attached to the sampler and pull it about 20 feet up and over the bridge railing. This was a fairly dangerous task that had to be rehearsed many times over. I feel that there were still changes being made to the sampler even between the on-site training I attended and when we received the sampler. At the training in Portland, we didn't actually get to see a working sampler in action. It almost feels that the USGS was the "guinea pig" or the "beta testers" for this sampler. What we really needed was a reliable system that produced a viable sample with consistency. We had 2 samplers and they each had their own little quirks. I realize and appreciate that the staff in Dr. Pankow's lab worked very hard and long on the SVAWS. However, I do not think this sampler was a good fit for what the project demanded.

While the samplers were easy to handle and install, I think that they need to be simplified and made a lot more rugged to withstand the rigors of deployment in the real world. Deploying the samplers in very flashy water subjected the samplers to a lot of pounding while in the PVC tubes. All that pounding can easily damage the inner workings of the samplers.

Regarding questions 1-2, the sampler was not difficult to operate or maintain. But learning curve was a little steep because of the short amount of time allowed to figure it out and, in my case, lack of training since I hadn't attended any (videos were helpful though). Regarding question 3, I scored it at 50 based on the costs that I saw. But I didn't have to pay for much since funds came from nawqa so hard for me to provide an informed opinion on this. Other random comments: I predicted that the filters would clog with sediment during runoff events that could have caused pressure problems with lines popping off etc. I was pleased to discover that never happened which makes me more optimistic that the sampler is useful in high turbidity deployments. I could make a much more informed judgement about the value of the sampler if I saw data. I hope that another evaluation of the sampler is taking place that takes into account important factors related to data quality. For example, sample holding time is a big concern. How is it that samples can sit in the sampler during the summer without refrigeration for a week and not be compromised? I think video training is effective and should be used to train future operators. Maybe revise a couple of them to be a little more focused on particular objectives. I thought the Pankow development and support team (you guys) were responsive to problems and provided timely and useful suggestions to address our problems, so that was appreciated. 